\title{
MIXED HODGE STRUCTURES
}

\author{
BY \\ FOUAD ELZEIN ${ }^{1}$
}

\begin{abstract}
The theory of Mixed Hodge Structures (M.H.S.) on the cohomology of an algebraic variety $X$ over complex numbers was found by Deligne in 1970 .

The case when $X$ is a Normal Crossing Divisor is fundamental. When the variety $X$ is embedded in a smooth ambient space we get the Mixed Hodge Structure using standard exact sequences in topology. This technique uses resolution of singularities one time for a complete variety and 2 times for a quasi-projective one.

As applications to the study of local cohomology we give the spectral sequence to the Mixed Hodge Structure on cohomology with support on a subspace $Y$.
\end{abstract}

Introduction. The theory of Mixed Hodge Structure (M.H.S.) on the cohomology of algebraic varieties was found by Deligne in 1970 [H II, H III]. The construction of Deligne consists of reducing to the case of the complement of Normal Crossing Divisors (N.C.D.) by using simplicial schemes and descent theorems on cohomology. We present a new construction of M.H.S. which consists of reducing to the N.C.D. case using desingularization and the fundamental exact sequence in cohomology between the cohomology with compact support in an open subset, the cohomology of the ambient space and the closed complement. In this way we avoid the construction of simplicial resolutions of varieties in order to obtain the M.H.S.; we need to apply the desingularization process one time for projective varieties, and two times for quasi-projective varieties.

The article is divided into two independent parts. In the first part we work directly on cohomology in as elementary a way as possible, and we need only the results of [H II]. In [H III] Deligne has introduced the concept of Mixed Hodge Complex (M.H.C.). In Part II we show that our methods give a construction of such complexes. We provide in this way many spectral sequences giving rise to M.H.S. In $\S 0$ we construct Mixed Cones of M.H.C. In §I we recall the fundamental and elementary cases after [H II] and [G]. In §II we lift the duality at spectral sequence level between M.H.S. of a N.C.D. and its complement to the complex level. This kind of construction is essential for our methods.

In §III, we give the basic constructions of M.H.C. in detail for projective varieties. Lemma III.1.2 shows how we obtain canonical M.H.S. from noncanonical constructions on complexes. We give complements to the proof in the Appendix.

I started working on this subject in Spring 1979 at Mittag-Leffler Institute, where Verdier told me that he is convinced that the M.H.S. on the cohomology of a variety

Received by the editors December 23, 1980. Presented to the 1981 AMS summer meeting at Arcata. 1980 Mathematics Subject Classification. Primary 14B05, 14B15, 14F99; Secondary 18E30.

'Supported by C.N.R.S. (ERA 589) and in part by a National Science Foundation grant. 
$X$ with support at an isolated singular point should depend on the normal bundle of the exceptional divisor in a desingularization of $X$. We study this question in the last paragraph, where we show the dependence on the normal bundle at the spectral level. The M.H.C. we construct depend only on an analytic neighborhood of the singularity. Discussions with Deligne and Verdier have been of great interest.

This paper is presented in such a way that we do not ask the reader to know about simplicial schemes. The idea of splitting it in two parts came to me after the deep and constructive criticism of the referee.

\section{Part I. Mixed Hodge Structures}

We assume here the construction given by Deligne in [H II] of the M.H.S. on a N.C.D. (see [G] also, and Part II of this paper, $\S \mathrm{I}$ ). Let $X$ be a smooth variety and $i$ : $Y \rightarrow X$ a closed embedding, $j: X-Y \rightarrow X$ the open embedding. We construct the M.H.S. on the cohomology of $Y$ using the exact sequence

$$
0 \rightarrow j_{*} \mathbf{Z}_{X-Y} \rightarrow \mathbf{Z}_{X} \rightarrow i_{*} \mathbf{Z}_{Y} \rightarrow 0 .
$$

To simplify proof and notations, we consider the proper case. Indications on the quasi-projective case will be given in Part II. There is no essential difference between the two cases.

M.H.S. for a complete variety.

1. Hypothesis. Suppose the variety $X$ above is proper, and consider the diagram

$$
\begin{array}{ccccc}
Y^{\prime} & \stackrel{i^{\prime}}{\rightarrow} & X^{\prime} & \stackrel{j^{\prime}}{\leftarrow} & X^{\prime}-Y^{\prime} \\
p_{Y} \downarrow & & \downarrow p & & \imath \downarrow p_{X-Y} \\
Y & \stackrel{i}{\rightarrow} & X & j & \\
& & X-Y
\end{array}
$$

where $X^{\prime}$ is a smooth variety, $p$ a proper morphism, $Y^{\prime}=p^{-1}(Y)$ is a N.C.D. in $X^{\prime}$, and the restriction $p_{X-Y}$ of $p$ is an isomorphism.

2. Proposition. With the previous notation, the trace map $\operatorname{Tr} p: \operatorname{R} p_{*} \mathbf{Z}_{X^{\prime}} \rightarrow \mathbf{Z}_{X}$ induces a map $\operatorname{Tr} p / Y: \mathbf{R} p_{*} \mathbf{Z}_{Y^{\prime}} \rightarrow \mathbf{Z}_{Y}$ which in turn induces maps $H^{*}\left(Y^{\prime}, \mathbf{Z}\right) \rightarrow$ $H^{*}(Y, \mathbf{Z}), H_{c}^{*}\left(Y^{\prime}, \mathbf{Z}\right) \rightarrow H_{c}^{*}(Y, \mathbf{Z})$. Those maps are necessarily compatible with any functorial mixed Hodge structure theory on cohomology.

Proof. On the cohomology level the trace morphism $H^{i}\left(X^{\prime}, \mathbf{Z}\right) \rightarrow H^{i}(X, \mathbf{Z})$ is obtained by Poincare isomorphisms from the morphisms in homology: $H_{2 n+2-i}\left(X^{\prime}, \mathbf{Z}\right) \rightarrow H_{2 n+2-i}(X, \mathbf{Z})$, where $\operatorname{dim} X=n+1$. In terms of derived categories the trace morphism $\operatorname{Tr} p: \mathbf{R} p_{*} p^{!} \mathbf{Z}_{X} \rightarrow \mathbf{Z}_{X}$ where $p^{!}$is the extraordinary inverse image, is defined in [V II, formula (2.3.4)]. A flabby resolution $\mathcal{F}^{\cdot}$ of $p^{!} \mathbf{Z}_{X}$ is given there, such that the trace is defined on the complex of sheaves $p_{*} \mathcal{F}^{\cdot}$ (here we have $\mathbf{Z}_{X^{\prime}} \stackrel{\approx}{\rightarrow} p^{!} \mathbf{Z}_{X}$ since $X^{\prime}$ is smooth). This trace morphism restricts to

$$
\operatorname{Tr} p / Y: \mathbf{R} p_{*} \mathbf{Z}_{Y^{\prime}} \cong p_{*} i^{* * \mathscr{F}} \cdot \rightarrow i^{*} \mathbf{Z}_{X} \simeq \mathbf{Z}_{Y}
$$

since $i^{*} \mathscr{F}^{\cdot}$ is flabby and $i^{*} p_{*}^{\mathscr{F}^{*}} \simeq p_{*} i^{*} \mathscr{F}^{\cdot}$. 
Compatibility with M.H.S. Suppose we have constructed a functorial M.H.S. as is done by Deligne, then we have the following diagram of M.H.S. where the rows are exact sequences (with coefficients in $\mathbf{C}$ ):

$$
\begin{aligned}
& H_{c}^{i}\left(X^{\prime}-Y^{\prime}\right) \stackrel{\mu_{i}^{\prime}}{\rightarrow} H^{i}\left(X^{\prime}\right) \quad \rightarrow \quad H^{i}\left(Y^{\prime}\right)
\end{aligned}
$$

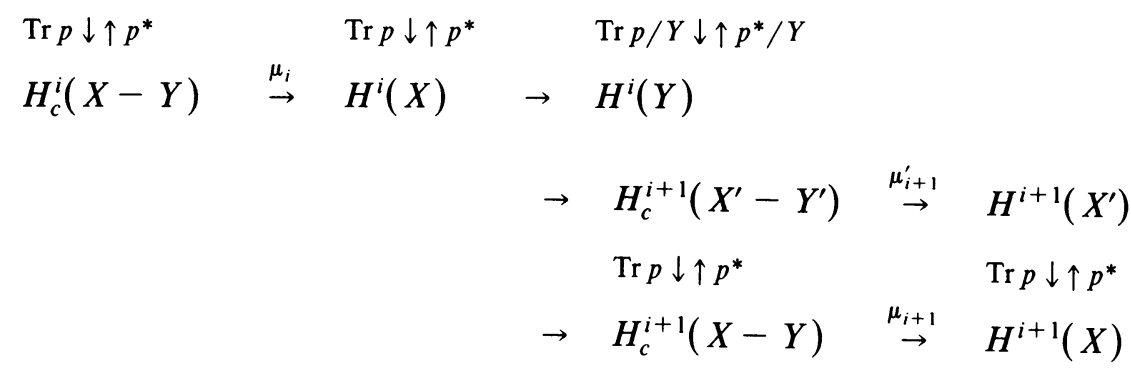

We know that $p^{*}$ is compatible with M.H.S. and we have $\operatorname{Tr} p \circ p^{*}=\mathrm{Id}$ and $\operatorname{Tr} p / Y \circ p^{*} / Y=\mathrm{Id}$. We want to show that $\operatorname{Tr} p / Y$ is compatible with M.H.S. It is immediately seen by duality that $\operatorname{Tr} p$ is compatible with M.H.S. on $X$ and $X-Y$. Since the category of M.H.S. is abelian, we may get short exact sequences with $L_{1}^{\prime}=\operatorname{coker} \mu_{i}^{\prime}, L_{1}=\operatorname{coker} \mu_{i}, L_{2}^{\prime}=\operatorname{ker} \mu_{i+1}^{\prime}, L_{2}=\operatorname{ker} \mu_{i+1}, H^{\prime}=H^{i}\left(Y^{\prime}\right)$ and $H=$ $H^{i}(Y)$.

$$
\begin{array}{ccccccccc}
0 & \rightarrow & L_{1}^{\prime} & \stackrel{\alpha^{\prime}}{\rightarrow} & H^{\prime} & \rightarrow & L_{2}^{\prime} & \rightarrow & 0 \\
& & \operatorname{Tr} p_{1} \downarrow \uparrow p_{1}^{*} & & \operatorname{Tr} p \downarrow \uparrow p^{*} & & \operatorname{Tr} p_{2} \downarrow 2 \uparrow p_{2}^{*} & & \\
0 & \rightarrow & L_{1} & \rightarrow & H & \rightarrow & L_{2} & \rightarrow & 0
\end{array}
$$

where $\operatorname{Tr} p_{i}$ is a morphism of M.H.S. such that $\operatorname{Tr} p_{i}{ }^{\circ} p_{i}^{*}=\mathrm{Id}$, and $\operatorname{Tr} p_{2}$ is the inverse of $p_{2}^{*}$. Let $C$ be the cokernel of $p_{1}^{*}$. The map $\operatorname{Tr} p_{1}$ defines a direct sum decomposition of $L_{1}^{\prime}$ into two sub-M.H.S. summands $C$ and $L_{1}$. Hence $\alpha^{\prime}(C)$ is a sub-M.H.S. object of $H^{\prime}$. Since $p_{2}^{*}$ is an isomorphism, $H^{\prime}$ is the direct sum of the two sub-M.H.S. objects $\alpha^{\prime}(C)$ and $H$, and $\operatorname{Tr} p$ is necessarily the corresponding projection of $H^{\prime}$ into $H$. Hence $\operatorname{Tr} p$ is compatible with M.H.S.

3. THEOREM. (i) With the notation of 1.1 , we have short exact sequences

$$
0 \rightarrow H^{i}\left(X^{\prime}, \mathrm{C}\right) \stackrel{i^{\prime *}-\operatorname{Tr} p}{\rightarrow} H^{i}\left(Y^{\prime}, \mathrm{C}\right) \oplus H^{i}(X, \mathrm{C}) \stackrel{\operatorname{Tr} p / Y+i^{*}}{\rightarrow} H^{i}(Y, \mathrm{C}) \rightarrow 0 .
$$

(ii) Suppose we have a canonical M.H.S. for projective N.C.D. (as in [H II]), then there exists a unique canonical M.H.S. on the cohomology of projective varieties such that the above sequences in ( $i$ ) are exact in the category of M.H.S.

Proof. (i) The exact sequence is deduced from the diagram (D) in the proof of Proposition 1.2. It is easy to check that the long exact sequence splits in short ones: the $\partial$ functor $H^{i}(Y) \rightarrow H^{i+1}\left(X^{\prime}\right)$ is the composition of the morphisms $H^{i}(Y) \rightarrow$ $H_{c}^{i+1}(X-Y) \rightarrow H^{i+1}(X) \rightarrow H^{i+1}\left(X^{\prime}\right)$ which is zero. We will study this exact sequence on the complex level later on. 
(ii) We define the M.H.S. on the cohomology of the variety $Y$ as the cokernel of the morphism $i^{\prime *}-\operatorname{Tr} p$ of M.H.S. of the smooth varieties $X^{\prime}$ and $X$, and the N.C.D. $Y^{\prime}$ in $X^{\prime}$. One can check the naturality of this construction and the independence of the choice of $X$ and $X^{\prime}$. Since it presents no particular difficulty we will admit it here. It is also reproduced on the complex level in the Appendix.

4. Remarks. (1) It may be more natural to view $H^{i}(Y, \mathrm{C})$ as a sub-M.H.S. of $H^{i}\left(Y^{\prime}, \mathrm{C}\right)$. The morphism $(\operatorname{Tr} p) / Y$ is a retraction.

(2) The theorem gives the uniqueness of the M.H.S. on the cohomology of $Y$, and so the M.H.S. here coincides with the one defined by Deligne.

(3) A way to view the morphism $(\operatorname{Tr} p) / Y$ is to consider neighborhood retracts $U^{\prime} \stackrel{p}{\rightarrow}$ of $Y^{\prime}$ and $Y$ and transport the trace $H^{*}\left(U^{\prime}\right) \rightarrow H^{*}(U)$ to $H^{*}\left(Y^{\prime}\right) \rightarrow H^{*}(Y)$ (as interpreted by $\mathrm{S}$. Bloch).

\section{Part II. Mixed Hodge Structures}

\section{The mixed category.}

1. We use the notation and terminology of [H II, H III].

This paragraph has been revised by J. L. Verdier.

1.1. Let $A$ be an abelian category. We denote by $F A$ (resp. $F_{2} A$ ) the category of filtered objects of $A$ (resp. bifiltered), with finite filtration, the morphisms respecting filtration; we denote by $C^{+} A$ (resp. $C^{+} F A, C^{+} F_{2} A$ ) the category of complexes of $A$ (resp. $F A, F_{2} A$ ) vanishing in degrees near $-\infty$, with morphisms of complexes. It is only in $K^{+} A$ (resp. $K^{+} F A, K^{+} F_{2} A$ ) that morphisms are considered up to homotopy. Finally $D^{+} A$ (resp. $D^{+} F A, D^{+} F_{2} A$ ) is the derived category [V, H III].

1.2. The mixed translate of a filtered complex and the mixed cone.

Definition 1.2.1. Let $(K, W)$ be a complex of objects of $A$ with an increasing filtration. The translation functor $T$ is denoted also by $K \rightarrow K[1,0]$. We define a translation functor $K \rightarrow K[0,1]=(K, W[1])$ which shifts the degree of $W$ such that $(W[1])_{n} K=W_{n-1} K$. The composite functor is denoted by $K \rightarrow K[1,1]=T_{M} K$.

REMARK. If there exists a decreasing filtration on $K$ as well, it does not shift.

Definition 1.2.2. Let $u: K \rightarrow K^{\prime}$ be a morphism in $C^{+} F A$ with increasing filtrations. The usual cone over $u$ is denoted by $C(u)$. The Mixed Cone $C_{M}(u)$ in $C^{+} F A$ is the complex $T_{M} K \oplus K^{\prime}$ with the usual differential [V]. Let $S_{K}: K[0,1] \rightarrow K$ be the canonical morphism, then $C_{M}()=C\left(u \circ S_{K}\right)$.

REMARK 1.2.3. Let $u$ and $u^{\prime}$ be two morphisms from $K$ to $K^{\prime}$, and $h$ a homotopy from $u$ to $u^{\prime}$. We have $u-u^{\prime}=d_{K^{\prime}} \circ h+h \circ d_{K}$. We define an isomorphism $I_{h}$ : $C(u) \stackrel{\sim}{\rightarrow} C\left(u^{\prime}\right)$ by the matrix $\left(\begin{array}{cc}\text { Id } & 0 \\ h & \text { Id }\end{array}\right)$ operating on $T K \oplus K^{\prime}$ (resp. filtered, bifiltered isomorphism). It is commutative with the injection of $K^{\prime}$ in $C(u)$ and $C\left(u^{\prime}\right)$, and with the projection on $T K$.

REMARK 1.2.4. Let $h$ and $h^{\prime}$ be two homotopies from $u$ to $u^{\prime}$. Then a second homotopy from $h$ to $h^{\prime}$, which is a family of morphisms $k^{i+2}: K^{i+2} \rightarrow K^{\prime i}(i \in \mathbf{Z})$ such that $h-h^{\prime}=d^{\prime} \circ k+k \circ d$, defines an homotopy between $I_{h}$ and $I_{h^{\prime}}$.

REMARK 1.2.5. Let $A$ be an abelian category, and suppose we are given morphisms $\varphi: X \rightarrow Y$ and $\varphi^{\prime}: X^{\prime} \rightarrow Y^{\prime}$ in $C^{+} A$ (resp. $C^{+} F A, C^{+} F_{2} A$ ) and a commutative diagram (I) in $D^{+} A$ (resp. $D^{+} F A, D^{+} F_{2} A$ ). 
(I)

$$
\begin{aligned}
& \begin{array}{l}
X \quad \stackrel{\varphi}{\rightarrow} Y \\
\end{array} \\
& \downarrow \alpha_{X} \quad \downarrow \alpha_{Y} \\
& X^{\prime} \stackrel{\varphi^{\prime}}{\rightarrow} Y^{\prime} \\
& \begin{array}{lllll}
Y & \rightarrow & C(\varphi) & \rightarrow & T X \\
\downarrow \alpha_{Y} & & \downarrow C\left(\alpha_{X}, \alpha_{Y}\right) & & \downarrow T \alpha_{X} \\
Y^{\prime} & \rightarrow & C\left(\varphi^{\prime}\right) & \rightarrow & T X^{\prime}
\end{array}
\end{aligned}
$$

Then there exists a noncanonical morphism $C\left(\alpha_{X}, \alpha_{Y}\right)$ in $D^{+} A$ (resp. $D^{+} F A$, $D^{+} F_{2} A$ ) which makes the diagram (II) commutative.

2. The category $C$ of Mixed Complexes (M.C.).

2.1. Let $\mathbf{Z}$ be the category of abelian groups. We defined $C^{+} \mathbf{Z}, D^{+} \mathbf{Z}$ and the corresponding filtered categories.

A M.C. $K$ is the following data ( $c f$. [H III]):

(i) $K_{\mathbf{Z}} \in$ ob $D^{+} \mathbf{Z}, K_{\mathbf{Q}} \in \mathrm{Ob} D^{+} F \mathbf{Q}, K_{\mathbf{C}} \in \mathrm{Ob} D^{+} F_{2} \mathbf{C}$.

(ii) $\alpha: K_{\mathbf{Z}} \otimes \mathbf{Q} \simeq K_{\mathbf{Q}}$ in $D^{+} \mathbf{Q}$ and $\beta:\left(K_{\mathbf{Q}}, W\right) \otimes \mathbf{C} \simeq\left(K_{\mathbf{C}}, W\right)$ in $D^{+} F \mathbf{C}$.

We do not put any Hodge type hypothesis on the M.C. for the moment. Such complexes form the objects of a category $C$. Over a topological space $X$, the cohomological M.C. (C.M.C.) is a sheafified version of M.C. where we take $K_{\mathbf{Z}}$ in $D^{+}(X, \mathbf{Z}), K_{\mathbf{Q}}$ in $D^{+}(X, W, \mathbf{Q})$ and $K_{\mathbf{C}}$ in $D^{+}(X, W, F, \mathbf{C})$. We have given the filtration the usual notation where $W$ is increasing and $F$ is decreasing.

2.2. We consider morphisms $u: K \rightarrow K^{\prime}$ of M.C. as follows:

(i) $u_{\mathbf{Z}}: K_{\mathbf{Z}} \rightarrow K_{\mathbf{Z}}^{\prime}$ in $D^{+} \mathbf{Z}, u_{\mathbf{Q}}: K_{\mathbf{Q}} \rightarrow K_{\mathbf{Q}}^{\prime}$ in $D^{+}(W, \mathbf{Q})$, $u_{\mathbf{C}}: K_{\mathbf{C}} \rightarrow K_{\mathbf{C}}^{\prime}$ in $D^{+}(W, F, \mathbf{C})$.

(ii) The following diagrams should be commutative:

$$
\begin{aligned}
& K_{\mathbf{Q}} \stackrel{u_{\mathbf{Q}}}{\rightarrow} \quad K_{\mathbf{Q}}^{\prime} \\
& \alpha \downarrow l \quad l \downarrow \alpha^{\prime} \quad \text { in } D^{+} \mathbf{Q} \text {, } \\
& K_{\mathbf{Z}} \otimes \mathbf{Q} \stackrel{u_{\mathbf{z}} \otimes \mathbf{Q}}{\rightarrow} K_{\mathbf{Z}}^{\prime} \otimes \mathbf{Q} \\
& K_{\mathbf{C}} \stackrel{u_{\mathbf{C}}}{\rightarrow} \quad K_{\mathbf{C}}^{\prime} \\
& \beta \downarrow) \quad\left\langle\downarrow \beta^{\prime} \quad \text { in } D^{+}(W, \mathbf{C})\right. \text {. } \\
& K_{\mathbf{Q}} \otimes \mathbf{C} \quad \stackrel{u_{\mathbf{Q}} \otimes \mathbf{C}}{\rightarrow} \quad K_{\mathbf{Q}}^{\prime} \otimes \mathbf{C}
\end{aligned}
$$

REMARK. We can always find a morphism $\tilde{u}: \tilde{K} \rightarrow K \underset{\rightarrow}{\approx} K^{\prime}$ such that $\tilde{u}_{\mathbf{Z}}, \tilde{u}_{\mathbf{Q}}, \tilde{u}_{\mathrm{C}}$ are defined in $C^{+} \mathbf{Z}, C^{+}(W, \mathbf{Q})$ and $C^{+}(W, F, \mathbf{C})$.

2.3. We define as usual a translation functor $T K$ also denoted by $K[1,0]$. A triangle in $C$ is distinguished if its image in $D^{+} \mathbf{Z}, D^{+}(W, \mathbf{Q})$ and $D^{+}(W, F, \mathbf{C})$ is distinguished. The category $C$ is triangulated in this way. 
2.4. We define as in 1.2 the shift on the weight filtration functor $K \rightarrow K[0,1]$. We have canonical morphisms $S_{K}: K[0,1] \rightarrow K$ in $C$. The mixed translation functor is defined by $T_{M} K=K[1,1]$.

2.5. Mixed triangles.

Definition. (i) A triangle $T$ in $C$ is called mixed
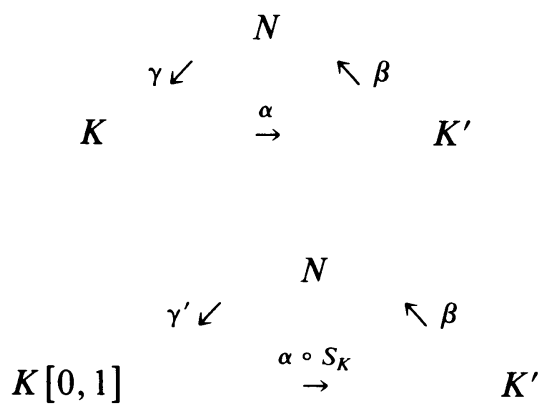

if the morphism $\gamma: N \rightarrow K[1,0]$ admits a factorisation $\gamma=\left(T S_{K}\right) \circ \gamma^{\prime}$ where $\gamma^{\prime}$ : $N \rightarrow K[1,1]$.

(ii) A mixed triangle $T$ is distinguished if its associated triangle $\left(\mathrm{T}^{\prime}\right)$ is isomorphic to a distinguished triangle in $C$.

REMARK. Distinguished mixed triangles in $C$ are not distinguished triangles. They are not in general stable by rotation. The composition of two consecutive maps in such a triangle is not necessarily zero.

2.6. Let $u: K \rightarrow K^{\prime}$ be a morphism of complexes. With the notation of 2.2 we may suppose $u_{\mathbf{Z}} \in C^{+} \mathbf{Z}, u_{\mathbf{Q}} \in C^{+}(W, \mathbf{Q})$ and $u_{\mathbf{C}} \in C^{+}(W, F, \mathbf{C})$ as in the remark. Then we define the mixed cone $C_{M}(u)$ as in 1.2:

(i) $C_{M}\left(u_{\mathbf{Z}}\right) \in D^{+} \mathbf{Z}, C_{M}\left(u_{\mathbf{Q}}\right) \in D^{+}(W, \mathbf{Q}), C_{M}\left(u_{\mathbf{C}}\right) \in D^{+}(W, F, \mathbf{C})$.

(ii) We choose $C_{M}\left(\alpha, \alpha^{\prime}\right): C_{M}\left(u_{\mathrm{Q}}\right) \simeq C_{M}\left(u_{\mathrm{z}}\right) \otimes \mathbf{Q}$ in $D^{+} \mathbf{Q}$ and $C_{M}\left(\beta, \beta^{\prime}\right)$ : $\left(C_{M}\left(u_{\mathrm{C}}\right), W\right) \simeq\left(C_{M}\left(u_{\mathrm{Q}}\right), W\right) \otimes \mathbf{C}$ in $D^{+} F \mathbf{C}$.

The cone $C_{M}(u)$ is not unique, but depends on the choice of $C_{M}\left(\alpha, \alpha^{\prime}\right)$ and $C_{M}\left(\beta, \beta^{\prime}\right)$. It provides a mixed distinguished triangle.

2.7. Let $T$ be a distinguished mixed triangle.

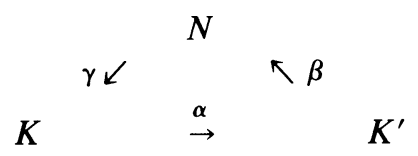

Then there exists a canonical isomorphism for any $n \in \mathbf{N}$,

$$
\mathrm{Gr}_{n}^{W} N \simeq \mathrm{Gr}_{n}^{W} K^{\prime} \oplus \mathrm{Gr}_{n-1}^{W} T K \text {. }
$$

Hence, for any cohomological functor, we have spectral sequences ${ }_{W} E_{r}^{p, q}$ for $K, K^{\prime}$ and $N$, such that

$$
{ }_{W} E_{\mathrm{l}}^{p, q}(N)=H^{p+q}\left(\mathrm{Gr}_{-p-1}^{W} T K \oplus \mathrm{Gr}_{-p}^{W} K^{\prime}\right)={ }_{W} E_{l}^{p+1, q} K \oplus{ }_{W} E^{p, q} K^{\prime} .
$$


For the first term only ${ }_{W} E_{\mathrm{i}}^{\cdot, q}(N)=C\left({ }_{W} E_{\mathrm{l}}, q(K) \stackrel{\alpha}{\rightarrow}{ }_{W} E_{\mathrm{i}},{ }^{, q}\left(K^{\prime}\right)\right)$ including differentials. We have a long exact sequence

$$
\cdots \rightarrow{ }_{W} E_{2}^{p, q}(K) \rightarrow{ }_{W} E_{2}^{p, q}\left(K^{\prime}\right) \rightarrow{ }_{W} E_{2}^{p, q}(N) \rightarrow{ }_{W} E_{2}^{p+1, q}(K) \rightarrow \cdots .
$$

3. The category $\mathfrak{R}$ C of M.C.

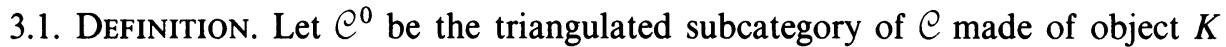
such that $K_{\mathbf{Z}}=0$ and $\left(K_{\mathrm{C}}, F\right)=0$.

The category $\Re \circlearrowright$ is the quotient of $e / e^{0}$.

REMARK. The category $\Re \circlearrowright e$ is triangulated. The weight shift becomes isomorphic to the identity in $\Re \mathcal{C}$ and the image in $\Re \mathcal{C}$ of distinguished mixed triangles of $C$ are distinguished in $\mathfrak{T} \mathcal{C}$. Over a topological space $X$, the functor "Hypercohomology of $X$ " factors through the cohomological $\mathfrak{A} \circlearrowright$. This hypercohomology is equipped with a Hodge filtration functorial on $\Re \mathcal{R}$.

As an example of elements in $\bigodot^{0}$, take $C_{M}(\mathrm{Id})$ the mixed cone over the identity on a M.C. $K$.

3.2. Definition. A mixed Hodge object, or in short, a Hodge object (C.M.H.C. in [H III]) is an object $K$ in $C$ such that

(a) $H^{*}\left(X, K_{\mathbf{z}}\right)$ is of finite type.

(b) For all $n,\left(H^{*}\left(X, \mathrm{Gr}_{n}^{W} K_{\mathbf{Q}}\right), H^{*}\left(X, \mathrm{Gr}_{n}^{W} K_{\mathrm{C}}\right)\right)$ satisfies the Hodge condition, i.e. the filtration $F$ and its conjugate give a Hodge decomposition.

RemarK. (1) If $K$ is Hodge, $K[0,1]$ and $K[1,0]$ are not necessarily Hodge but $K[1,1]$ is.

(2) In a distinguished mixed triangle, if two of the objects are Hodge, so is the third.

3.3. Deligne's fundamental theOREM. If $K$ is a Hodge object in $C$ then $H^{*}(X, K)$ is equipped with a M.H.S. The spectral sequence with respect to the $W$-filtration degenerates at the $E_{2}$ level, and with respect to the $F$ filtration at the $E_{1}$ level.

3.4. Let $T$ be, as in 2.7 , a mixed distinguished triangle in $C$ of Hodge objects. This triangle gives rise to a long sequence

$$
\rightarrow K[n, n] \stackrel{(-1)^{n} \alpha[n, n]}{\rightarrow} L[n, n] \stackrel{(-1)^{n} \beta[n, n]}{\rightarrow} N[n, n] \stackrel{(-1)^{n} \gamma[n, n]}{\rightarrow} K[n+1, n+1] \rightarrow \cdots .
$$

Taking hypercohomology we get an exact sequence of M.H.S.

$$
\begin{aligned}
\ldots H^{n}(X, K)[0, n] \stackrel{\alpha}{\rightarrow} H^{n}(X, L)[0, n] \stackrel{\beta}{\rightarrow} H^{n}(X, N)[0, n] \\
\stackrel{\gamma}{\rightarrow} H^{n+1}(X, K)[0, n+1] .
\end{aligned}
$$

Convention. Denote $\bar{W}$ the filtration on hypercohomology deduced from the $W$-filtration on complexes. Notice the shift on this $\bar{W}$ filtration. Define the weight filtration $W$ on the hypercohomology of $K$ as $W=\bar{W}[m]$ on $H^{m}(X, K)(\bar{W}$ shifts $m$ times to the right because it is increasing: $\left.W_{l}=\bar{W}_{l-m}\right)$. Then, in the long exact sequence the shift does not occur anymore. 
COROLlary. With the convention above, we have in Deligne's theorem

$$
\mathrm{Gr}_{n}^{W} H^{m}(X, K)={ }_{W} E_{2}^{m-n, n} .
$$

3.5. Denote by $\operatorname{Hdg}(X)$ the full subcategory of $\Re \bigodot$ generated by Hodge objects. There exist on $H^{*}(X, K)$ a M.H.S. functorial in $K \in \operatorname{Hdg}(X)$.

3.6. Deligne constructed in [H III] bifiltered resolutions of C. Dubois [D] has shown that the filtration $F$ is unique up to quasi-isomorphism, however the resolutions are not unique up to isomorphisms in the category $C$. One may raise the question whether they are unique in the category $\mathfrak{R}$, , up to isomorphisms in $\mathfrak{T}$.

I. The fundamental simplicial resolutions. The general construction of the Mixed Hodge Structure (M.H.S.) use simplicial resolutions. We will need in this work the elementary cases only, which are, however, fundamental. We recall here a brief description of these cases without resuming the proofs in Deligne [H II, H III] and Griffith-Schmid [G]. In fact, all are derived from Mayer-Vietoris resolutions.

1. M.H.S. of a Normal Crossing Divisor (N.C.D.). Let $X$ be a smooth variety, projective over C, and $Y$ a N.C.D. with smooth irreducible components $\left(Y_{i}\right)_{i \in I}$ of dimension $n$. Let $S_{q}$ be the set of strictly increasing sequences $\left(i_{0}, \ldots, i_{q}\right)$ in $I$, and $Y^{(q)}=\amalg_{S_{q}} Y_{i_{0}} \cap \cdots \cap Y_{i_{q}}$ the disjoint sum of the intersections of $Y_{i}$. Let $\Pi: Y^{(q)} \rightarrow Y$ be the finite projection and $\lambda_{j, q}: Y_{i_{0}} \cap \cdots \cap Y_{i_{q}} \rightarrow Y_{i_{0}} \cap \cdots \hat{i}_{j} \cdots \cap Y_{i_{q}}$ the closed embedding for $j \leqslant q$.

Definition. The Mayer-Vietoris simplicial resolution of $\mathbf{Q}_{Y}$ is the following complex of sheaves, denoted by $\Pi_{*} \mathbf{Q}_{Y(\cdot)}$ or simply $\mathbf{Q}_{Y(\cdot)}$.

$$
0 \rightarrow \Pi_{*} \mathbf{Q}_{Y(0)} \rightarrow \Pi_{*} \mathbf{Q}_{Y(1)} \rightarrow \cdots \rightarrow \Pi_{*} \mathbf{Q}_{Y(q-1)} \rightarrow \Pi_{d_{q-1}} \mathbf{Q}_{Y(q)} \rightarrow \cdots
$$

where $d_{q-1}=\sum_{j=0}^{q}(-1)^{j} \lambda_{j, q}^{*}$. The weight filtration $W$ is defined by

$$
W_{-q}\left(\mathbf{Q}_{Y(\cdot)}\right)=\bigoplus_{m \geqslant q} \Pi_{*} \mathbf{Q}_{Y(m)} .
$$

We have $\mathrm{Gr}_{-q}^{W} \mathbf{Q}_{Y(\cdot)} \simeq \Pi_{*} \mathbf{Q}_{Y(q)}[-q]$. The spectral sequence with respect to $W$ degenerates at rank 2 and is given by

$$
{ }_{W} E_{\mathrm{I}}^{p, q}=H^{p+q}\left(Y, \mathrm{Gr}_{-p}^{W}\left(\mathbf{Q}_{Y(\cdot)}\right)\right)=H^{p+q}\left(Y, \Pi_{*} \mathbf{Q}_{Y(p)}[-p]\right)=H^{q}\left(Y^{(p)}, \mathbf{Q}\right) .
$$

The differential $\delta: E_{\uparrow}^{p, q} \rightarrow E_{\uparrow}^{p+1, q}$, is given by

$$
\boldsymbol{\delta}=\sum_{j \leqslant p+1}(-1)^{j} \lambda_{j, p+1}^{*}: H^{q}\left(Y^{(p)}, \mathbf{Q}\right) \rightarrow H^{q}\left(Y^{(p+1)}, \mathbf{Q}\right) .
$$

In the same way we have the resolution $\Omega_{Y(\cdot)}^{*}$ of $\mathbf{C}_{Y}$ :

$$
0 \rightarrow \Pi_{*} \Omega_{Y(0)}^{*} \rightarrow \Pi_{*} \Omega_{Y(1)}^{*} \rightarrow \cdots \rightarrow \Pi_{*} \Omega_{Y(q)}^{*} \rightarrow \cdots
$$

with weight and Hodge filtrations. 
We represent ${ }_{W} E_{\mathrm{i}}^{\cdot \bullet}$ as follows:

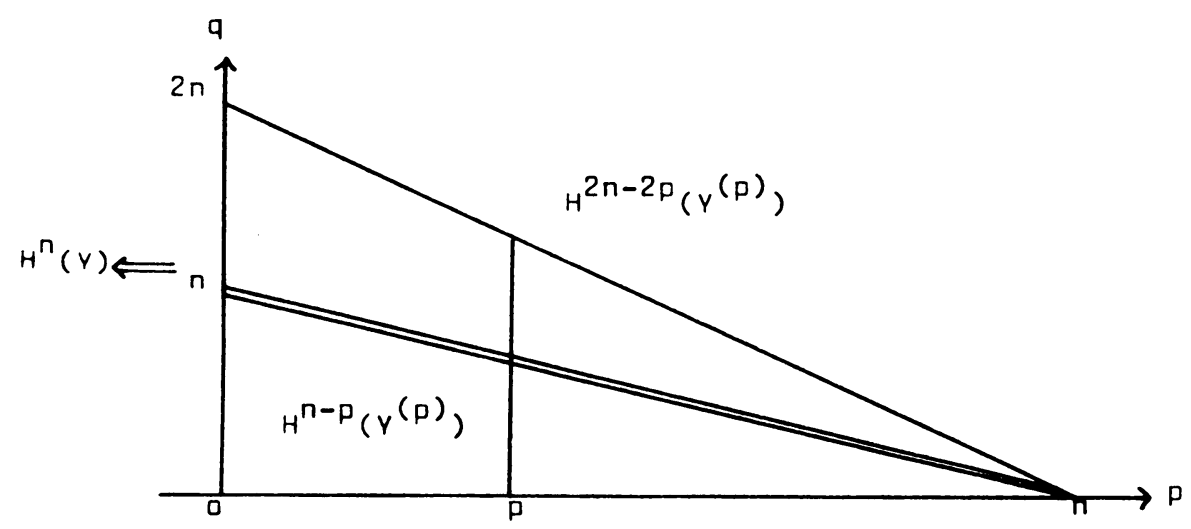

$E^{p, q}$ is 0 outside the triangle. The terms on the double line converge to $H^{n}(Y)$. The weight on $H^{n}(Y)$ is given by $q$ and varies from 0 to $n$.

2. M.H.S. on a smooth variety: $H^{*}(X-Y)$. We are concerned now by the M.H.S. on $X-Y$, the complement of the N.C.D. in the previous case. Set $X=X^{(0)}=Y^{(-1)}$ and $X^{(m)}=Y^{(m-1)}$ for $m \geqslant 1$. Deligne [H II] constructed the logarithmic complex $\Omega_{X}^{*}(\log Y)$ or $\Omega_{X}^{*}\langle Y\rangle$ with weight and Hodge filtrations $W$. and $F^{*}$. The weight filtration on rational cohomology is deduced from the spectral sequence with respect to the $\tau$ filtration on $\mathbf{R} j_{*} \mathbf{Q}_{X-Y}$ where $j: X-Y \rightarrow X$ is the open embedding. The spectral sequence with respect to $W$ degenerates at rank 2 , and is given by

$$
{ }_{W} E_{1}^{p, q}=H^{p+q}\left(X, \mathrm{Gr}_{-p}^{W} \Omega_{X}^{*}(\log Y)\right) \Rightarrow \mathrm{Gr}_{-p}^{W} H^{p+q}(X-Y) .
$$

In fact, the weight on $H^{p+q}(X-Y)$ that we are interested in, is the one deduced from the spectral sequence shifted by $p+q$ to the right. So, using the fact

$$
\operatorname{Res}: \operatorname{Gr}_{-p}^{W}\left(\Omega_{X}^{*}(\log Y)\right) \simeq \Pi_{*} \Omega_{Y^{(-p-1)}}^{*}[p]
$$

we have

$$
\begin{gathered}
{ }_{W} E_{\mathrm{l}}^{p, q} \simeq H^{2 p+q}\left(Y^{(-p-1)}, \mathbf{C}\right) \otimes \mathbf{C}(p) \Rightarrow \mathrm{Gr}_{q}^{W} H^{p+q}(X-Y) . \\
\text { weight } q
\end{gathered}
$$

The differential

$$
\delta: H^{2 p+q}\left(Y^{(-p-1)}, \mathbf{C}\right) \otimes \mathbf{C}(p) \rightarrow H^{2 p+q+2}\left(Y^{(-p-2)}, \mathbf{C}\right) \otimes \mathbf{C}(p+1)
$$

is deduced from Gysin morphisms of the embedding $Y^{(-p-1)} \rightarrow Y^{(-p-2)}$. 


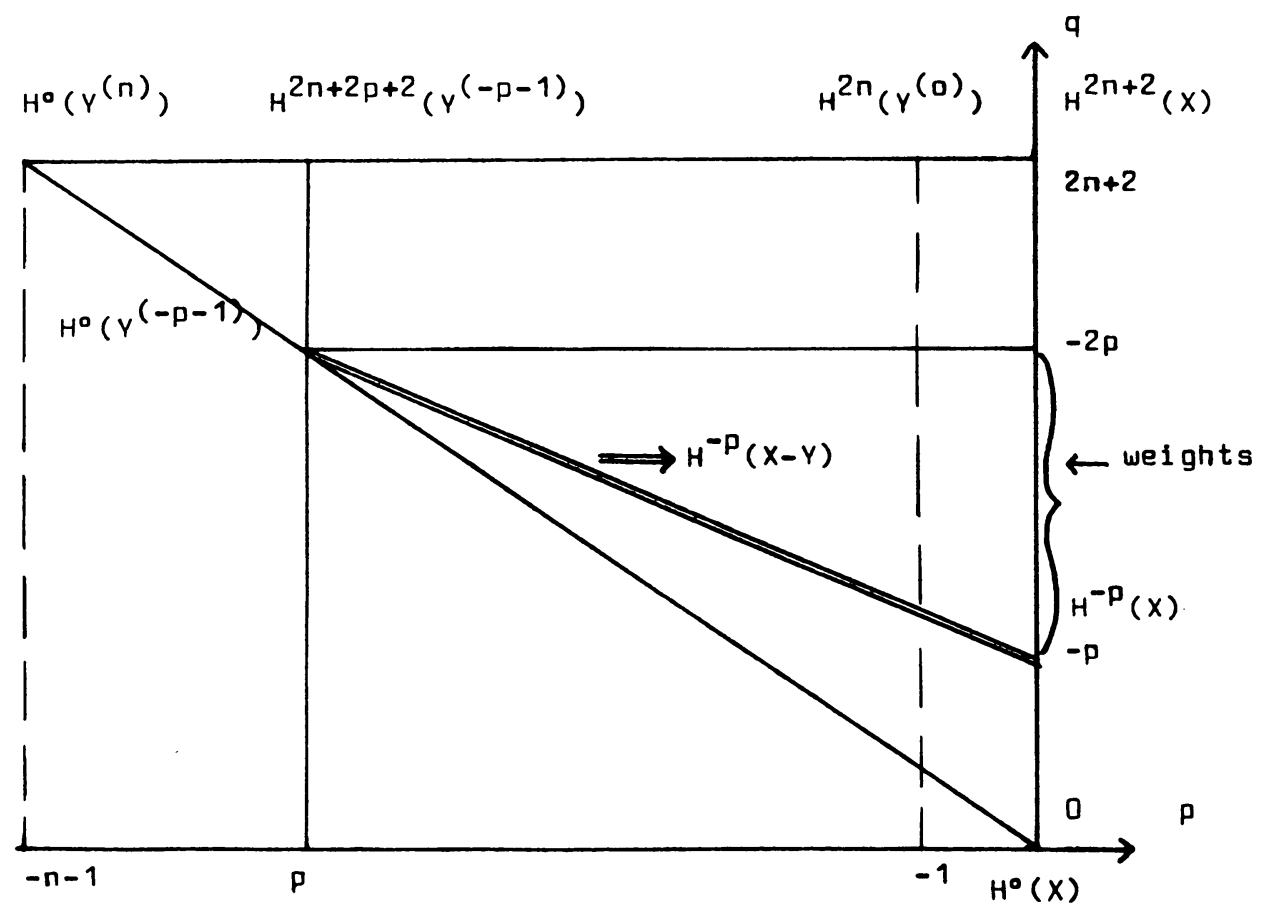

${ }_{W} E_{1}^{p, q}=0$ outside of the triangle. The terms on the double line converge to $H^{-p}(X-Y)$ with weight varying from $-p$ to $-2 p$.

3. M.H.S. on the cohomology with compact support of a smooth variety: $H_{c}^{*}(X-Y)$. With the previous notation in (1), denote by $\mathbf{Q}_{X-Y(\cdot)}$ the complex

$$
\mathbf{Q}_{X} \rightarrow \mathbf{Q}_{Y(\cdot)}[-1,-1] \text {. }
$$

It is a cone over the morphism $\mathbf{Q}_{X} \rightarrow \mathbf{Q}_{Y(0)}$ in degree 1, so that

$$
\mathbf{Q}_{X-Y(\cdot)} \simeq C_{M}\left[\mathbf{Q}_{X}[-1,-1] \rightarrow \mathbf{Q}_{Y(\cdot)}[-1,-1]\right] .
$$

We have the quasi-isomorphism $j_{!} \mathbf{Q}_{X-Y} \approx \mathbf{Q}_{X-Y(\cdot)}$, and a weight filtration on $\mathbf{Q}_{X-Y(\cdot)}$, with associated spectral sequence:

$$
\begin{aligned}
& { }_{W} E_{1}^{0, q}=H^{q}\left(X, \mathrm{Gr}_{0}^{W} \mathbf{Q}_{X-Y(\cdot)}\right) \simeq H^{q}(X, \mathbf{Q}), \\
& { }_{W} E_{1}^{p, q}=H^{p+q}\left(X, \mathrm{Gr}_{-p}^{W} \mathbf{Q}_{X-Y(\cdot)}\right) \simeq H^{q}\left(Y^{(p-1)}, \mathbf{Q}\right) \text { for } p \geqslant 1 .
\end{aligned}
$$

The differential $\delta: H^{q}(X, \mathbf{Q}) \rightarrow H^{q}\left(Y^{(0)}, \mathbf{Q}\right)$ is $\Sigma(-1)^{j} i_{j}^{*}$ for $i_{j}: Y_{j} \rightarrow X$. The weights of $H_{c}^{m}(X-Y, \mathbf{Q})$ vary from 0 to $m$. We define in the same way the bifiltered complex $\Omega_{X-Y(\cdot)}^{*}$. We get from the short exact sequence: $0 \rightarrow \Omega_{Y(\cdot)}^{*}[-1,-1] \rightarrow$ $\Omega_{X-Y(\cdot)}^{*} \rightarrow \Omega_{X}^{*} \rightarrow 0$, the long exact sequence of M.H.S.

$$
\rightarrow H^{i-1}(Y, \mathrm{C}) \rightarrow H_{c}^{i}(X-Y, \mathrm{C}) \rightarrow H^{i}(X, \mathrm{C}) \rightarrow H^{i}(Y, \mathrm{C}) \rightarrow H_{c}^{i+1}(X-Y, \mathrm{C}) \ldots
$$

4. M.H.S. on the cohomology with support on a N.C.D.: $H_{Y}^{*}(X)$. Consider the quotient complex $\Omega_{X}^{*}\langle Y\rangle / \Omega_{X}^{*}$ with the image weight and Hodge filtrations. The weight filtration can be defined on rational cohomology; it comes from the $\tau$ 
filtration on $\mathbf{R} \Gamma_{Y}(X, \mathbf{Q})$. We have the quasi-isomorphisms

$$
\mathbf{R} \Gamma_{Y}\left(X, \Omega_{X}^{*}\langle Y\rangle / \Omega_{X}^{*}\right) \simeq \mathbf{R} \Gamma_{Y}(X, \mathbf{C})[1] .
$$

Then, the mixed translate $\Omega_{X}^{*}\langle Y\rangle / \Omega_{X}^{*}[-1,-1]$ is a C.M.H.C. (cohomological mixed Hodge complex) which gives the M.H.S. of $H_{Y}^{*}(X, \mathrm{C})$. We have

$$
\begin{gathered}
\mathrm{Gr}_{i}^{W}\left(T_{M}^{-1} \Omega_{X}^{*}\langle Y\rangle / \Omega_{X}^{*}\right) \simeq T^{-1} \mathrm{Gr}_{i+1}^{W}\left(\Omega_{X}^{*}\langle Y\rangle / \Omega_{X}^{*}\right) \simeq \Pi_{*} \Omega_{Y(i)}^{*}[-i-2], \\
{ }_{W} E_{1}^{p, q}=H^{2 p+q-2}\left(Y^{(-p)}, \mathrm{C}\right), \quad{ }_{W} E_{1}^{p, q}=0 \quad \text { for } p \notin[-n, 0] .
\end{gathered}
$$

We have a long exact sequence of M.H.S.

$$
\rightarrow H_{Y}^{m}(X, C) \rightarrow H^{m}(X, C) \rightarrow H^{m}(X-Y, C) \rightarrow H_{Y}^{m+1}(X, C) \rightarrow \cdots .
$$

The weights of $H_{Y}^{m}(X, C)$ vary from $m$ to $2 m-2$.

REMARK 1 . One can also use the complex $C_{M}\left(\Omega_{X}^{*}[-1,-1] \rightarrow \Omega_{X}^{*}\langle Y\rangle[-1,-1]\right)$ to get a M.H.S. on $H_{Y}^{*}(X)$. It is not bifiltered quasi-isomorphic to the previous one. This means the terms ${ }_{W} E_{1}^{p, q}$ differ. We get however the same M.H.S. as we can see from the existence of a natural morphism into $\Omega_{X}^{*}\langle Y\rangle / \Omega_{X}^{*}[-1,-1]$ which respect the filtrations, inducing an isomorphism on the hypercohomology which respect the filtrations. Then the morphism is necessarily an isomorphism of M.H.S. This means that the terms ${ }_{W} E_{2}^{p, q}$ coincide, as one can check easily.

REMARK 2. $C\left(\Omega_{X}^{*}[-1,-1] \rightarrow \Omega_{X}^{*}\langle Y\rangle[-1,-1]\right)$ is quasi-isomorphic in $D^{+} \mathscr{F}_{2}(X, C)$ to $\Omega_{X}^{*}\langle Y\rangle / \Omega_{X}^{*}[-1,-1]$.

5. Open N.C.D. Consider a partition $I=I_{0} \cup I_{1}$ of the index set of the irreducible components of the N.C.D. $Y$ and set $Z=\cup_{i \in I_{0}} Y_{i}$ a sub-N.C.D. of $Y$. We want to construct a M.H.S. on the cohomology of $Y-Z$.

The closure $T=\overline{(Y-Z)}=\cup_{i \in I_{1}} Y_{i}$ of $Y-Z$ is a N.C.D. in $X$. Consider the simplicial scheme $\Pi^{(\cdot)}: T^{(\cdot)} \rightarrow T$ constructed as in (1) above, and write $T^{(\cdot)}-Z$ for $T^{(\cdot)}-\Pi^{(\cdot)-1}(Z)$ which is the simplicial resolution of $T-Z$.

$$
\begin{array}{lll}
T^{(\cdot)}-Z & \stackrel{j^{(\cdot)}}{\rightarrow} & T^{(\cdot)} \\
\Pi^{(\cdot)} \downarrow & & \downarrow \Pi^{(\cdot)} \\
T-Z & \stackrel{j}{\rightarrow} & T
\end{array}
$$

(Notation. In the following, $\Pi_{*}^{(\cdot)}$ already contains the direct sum passing from a double complex to a simple one.) We have the quasi-isomorphism $\mathbf{R} j_{*} \mathbf{Q}_{T-Z} \approx$ $\Pi_{*}^{(\cdot)} \mathbf{R} j_{*}^{(\cdot)} \mathbf{Q}_{T(\cdot)-Z}$ in $D^{+}(T, \mathbf{Q})$. Denote by $K_{Y-Z}^{\mathbf{Q}}$ the complex above, and more precisely a particular choice of a complex representing it, using Godement resolutions for example.

The C.M.H.C. of $Y-Z$ is defined as follows:

(i) The complex $\mathbf{R} j_{*} \mathbf{Z}_{Y-Z} \in \mathrm{Ob} D^{+}(T, \mathbf{Z})$.

(ii) The complex $K_{Y-Z}^{\mathbf{Q}}$ with the following increasing filtration $W$ :

Consider for each $q \in \mathbf{N}$ the filtration $\tau_{\leqslant n}[\mathbf{H}$ II $]$ on $\mathbf{R}_{*}^{j_{*}^{(q)}} \mathbf{Q}_{T^{(q)}-7}$. Then define $W$ as the diagonal filtration [H III]

$$
W_{n}=\bigoplus_{q} \tau_{\leqslant n+q} \Pi_{*}^{(q)} \mathbf{R} j_{*}^{(q)} \mathbf{Q}_{T^{(q)}-Z}
$$


(iii) The complex $K_{Y-Z}^{\mathrm{C}}=\Pi_{*}^{(\cdot)} \Omega_{T^{(\cdot)}}^{*}\langle Z\rangle$ with a filtration $W$ constructed as above, and a Hodge filtration [H III].

(iv) Quasi-isomorphisms: $\mathbf{C} \otimes\left(K_{Y-Z}^{\mathbf{Q}}, W\right) \cdots\left(K_{Y-Z}^{\mathbf{C}}, W\right)$ in $D^{+} F(Y-Z, \mathbf{C})$ and $Q \otimes \mathbf{R} j_{*} \mathbf{Z}_{Y-Z} \simeq K_{Y-Z}^{\mathbf{Q}}$ in $D^{+}(Y-Z, \mathbf{Q})$.

Notation. We also use $\Omega_{Y^{(\cdot)}}^{*}\langle Z\rangle$ to denote this complex. We have

$$
\begin{aligned}
\mathrm{Gr}_{n}^{W}\left(K_{Y-Z}^{\mathbf{Q}}\right) & \simeq \bigoplus_{p} \mathrm{Gr}_{n+p}^{\tau}\left(\Pi_{*}^{(p)} \mathbf{R} j_{*}^{(p)} \mathbf{Q}_{T^{(p)}-Z}\right)[-p] \\
& \simeq \bigoplus_{p} \Pi_{*}^{(p)} \mathbf{R}^{n+p} j_{*}^{(p)} \mathbf{Q}_{T^{(p)}-Z}[-n-2 p] .
\end{aligned}
$$

The associated spectral sequence is

$$
{ }_{W} E_{1}^{p, q}=H^{p+q}\left(T, \mathrm{Gr}_{p}^{W} K_{Y-Z}^{\mathbf{Q}}\right) \simeq \bigoplus_{r} H^{p+q}\left(T^{(r)}, \mathbf{R}^{r-p} j_{*}^{(r)} \mathbf{Q}_{T^{(r)}-Z}[p-2 r]\right) .
$$

Let $Z_{r}^{(\cdot)}$ be the simplicial resolution of the N.C.D. $T^{(r)} \cap\left(\Pi^{(r)^{-1}} Z\right)$ in $T^{(r)}$. Then $\mathbf{R}^{m} j_{*}^{(r)} \mathbf{Q}_{T^{(r)}-Z} \simeq \Pi_{r, *}^{(m-1)} \mathbf{Q}$ where $\Pi_{r}^{(m-1)}: Z_{r}^{(m-1)} \rightarrow T^{(r)}$ is the projection. Finally we have

$$
{ }_{W} E_{1}^{p \cdot q} \simeq \bigoplus_{r} H^{2 p+q-2 r}\left(Z_{r}^{(r-p-1)}, \mathbf{Q}\right) .
$$

6. Functoriality for Mayer-Vietoris resolutions. Let

$$
\begin{array}{lllll}
Z^{\prime} & \hookrightarrow & Y^{\prime} & \hookrightarrow & X^{\prime} \\
\downarrow & & \downarrow f & & \downarrow f^{\prime} \\
Z & \hookrightarrow & Y & \hookrightarrow & X
\end{array}
$$

be a diagram where $f^{\prime}$ is a morphism of smooth varieties inducing morphisms $f$ on the N.C.D. $Y^{\prime}$ and on the sub-N.C.D. $Z^{\prime}$. We can define extensions respecting filtrations:

(i) $f^{*}: \mathbf{Q}_{Y^{(\cdot)}} \rightarrow f_{*} \mathbf{Q}_{Y^{(\cdot)}}$ of $f^{*}: \mathbf{Q}_{Y} \rightarrow f_{*} \mathbf{Q}_{Y^{\prime}}$,

$f^{*}: \Omega_{Y^{(\cdot)}}^{*} \rightarrow f_{*} \Omega_{Y^{\prime}(\cdot)}^{*}$ of $f^{*}: \mathbf{C}_{Y} \rightarrow f_{*} \mathbf{C}_{Y^{\prime}}$,

(ii) $f^{*}: K_{Y-Z}^{\mathbf{Q}} \rightarrow \mathbf{R} f_{*} K_{Y^{\prime}-Z^{\prime}}^{\mathbf{Q}}$ of $f^{*}: \mathbf{Q}_{Y-Z} \rightarrow f_{*} \mathbf{Q}_{Y^{\prime}-Z^{\prime}}$,

$f^{*}: K_{Y-Z}^{\mathbf{C}} \rightarrow \mathbf{R} f_{*} K_{Y^{\prime}-Z^{\prime}}^{\mathbf{C}}$ of $f^{*}: \mathbf{C}_{Y-Z} \rightarrow f_{*} \mathbf{C}_{Y^{\prime}-Z^{*}}$.

(In (ii) one can imagine defining $\mathbf{R} f_{*}$ by Godement resolutions.)

We give the details for the first case.

Let $\left(Y_{i}^{\prime}\right)_{i \in I}$ and $\left(Y_{j}\right)_{j \in J}$ be the irreducible and smooth components of $Y^{\prime}$ and $Y$. We define the following partition of $I=\bigcup_{j \in J} I_{j}$ :

$$
\begin{aligned}
& I_{0}=\left\{i \in I: f\left(Y_{i}^{\prime}\right) \subset Y_{0}\right\}, I_{1}=\left\{i \in I-I_{0}: f\left(Y_{i}^{\prime}\right) \subset Y_{1}\right\}, \ldots, \\
& \qquad I_{j}=\left\{i \in I-\bigcup_{k \in[1, j-1]} I_{k}: f\left(Y_{i}^{\prime}\right) \subset Y_{j}\right\},
\end{aligned}
$$

and define $f_{q}^{*}$ for a section $m$ of $\Pi_{*}^{q} \mathbf{Q}_{Y_{j_{0}} \cap \cdots \cap Y_{j_{q}}}$ by

$$
f_{q}^{*}(m)=\prod_{i_{0} \times \cdots \times i_{q} \in J_{j_{0}} \times \cdots \times J_{j_{q}}}\left(f^{*} m / Y_{i_{0}} \cap \cdots \cap Y_{i_{q}}\right),
$$

a section of

$$
\prod_{i_{0} \times \cdots \times i_{q} \in J_{j_{0}} \times \cdots \times J_{j_{q}}} f_{*} \Pi_{*}^{(q)} \mathbf{Q}_{Y_{i_{0}}^{\prime} \cap \cdots \cap Y_{i_{q}}^{\prime}} \subset f_{*} \Pi_{*}^{(q)} \mathbf{Q}_{Y^{\prime(q)}}
$$


II. Duality. In order to construct the M.H.S. of a N.C.D. $Y$ embedded in $X$ which is smooth and proper, we used Mayer-Vietoris resolution, and for the complementary $X-Y$ we used the logarithmic complex $\Omega_{X}^{*}\langle Y\rangle$. The spectral sequences with respect to the weight filtration in either case are dual. For our purpose, we need to lift this duality to the M.H.C. level.

0 . Dual filtrations.

1. Let $(K, W)$ and $\left(K^{\prime}, W^{\prime}\right)$ be two complexes in $C^{+} F A$, with increasing (resp. decreasing) filtrations. We define on $H=\operatorname{Hom}\left(K, K^{\prime}\right)$ an increasing (resp. decreasing) filtration $W$, functorial in $K$ and $K^{\prime}$, by the formula

$$
W_{r} H=\left\{f \in H \text { such that for any } n \in \mathbf{Z}, f\left(W_{n} K\right) \subset W_{n+r}^{\prime} K^{\prime}\right\} .
$$

2. Suppose the filtration $W^{\prime}$ is trivial, i.e. $W_{i}^{\prime}=0$ for $i<l$ and $W_{i}^{\prime}=K^{\prime}$ for $i \geqslant l$. Then

$$
W_{r} H=\operatorname{ker}\left(H \rightarrow \operatorname{Hom}\left(W_{l-r-1} K, K^{\prime}\right)\right) \simeq \operatorname{Hom}\left(K / W_{l-r-1} K, K^{\prime}\right) .
$$

We want to prove with this hypothesis on $W^{\prime}\left(K^{\prime}\right)$, that the notion of dual filtration is well defined on $\mathbf{R} \operatorname{Hom}\left(K, K^{\prime}\right)$ in $D^{+} F A$, and we have

$$
\operatorname{Gr}_{n}^{W} \mathbf{R} \operatorname{Hom}\left(K, K^{\prime}\right) \simeq \mathbf{R} \operatorname{Hom}\left(\mathrm{Gr}_{l-n}^{W} K, K^{\prime}\right) .
$$

Suppose $K^{\prime}$ is a complex of injective objects in $F A$, i.e. injective objects in $A$ in our case. From the short exact sequence: $0 \rightarrow W_{l-n-1} K \rightarrow W_{l-n} K \rightarrow \mathrm{Gr}_{l-n}^{W} K \rightarrow 0$ we get dually

$$
0 \rightarrow \operatorname{Hom}\left(\mathrm{Gr}_{l-n}^{W} K, K^{\prime}\right) \rightarrow \operatorname{Hom}\left(W_{l-n} K, K^{\prime}\right) \rightarrow \operatorname{Hom}\left(W_{l-n-1} K, K^{\prime}\right) \rightarrow 0
$$

and finally

$$
0 \rightarrow \operatorname{Hom}\left(\mathrm{Gr}_{l-n}^{W} K, K^{\prime}\right) \rightarrow H / W_{n-1} H \rightarrow H / W_{n} H \rightarrow 0
$$

or

$$
\mathrm{Gr}_{n}^{W} H \simeq \operatorname{Hom}\left(\mathrm{Gr}_{l-n}^{W} K, K^{\prime}\right)
$$

Remarks. (1) We have $\operatorname{Hom}\left(K, K^{\prime}\right)[1,1] \simeq \operatorname{Hom}\left(K[-1,-1], K^{\prime}\right)$.

(2) Let $u: K_{1} \rightarrow K_{2}$ be a filtered morphism. Then

$$
\operatorname{Hom}\left(C_{M}(u), K^{\prime}\right) \simeq T_{M}^{-1} C_{M}\left(\operatorname{Hom}\left(K_{2}, K^{\prime}\right) \rightarrow \operatorname{Hom}\left(K_{1}, K^{\prime}\right) .\right.
$$

1. Dual logarithmic complex.

1. Let $K_{X}^{\cdot}$ denote the dualizing complex [RD] for the smooth variety $X$ of $\operatorname{dim} n+1$. We want to define a total differential $\Delta_{X-Y}$ on the graded group

$$
D^{\bullet}\langle X-Y\rangle=\underline{\operatorname{Hom}}_{X}\left(\Omega_{X}^{*}\langle Y\rangle, K_{X}^{*}\right) \text {. }
$$

The difficulty comes from the fact that the differential $d$ on $\Omega_{X}^{*}$ is not $\theta_{X}$-linear. To motivate the definition of $\Delta_{X-Y}$, we introduce a new complex.

2. The differential. Consider

$$
D_{\Omega^{*}}^{\cdot}\langle X-Y\rangle=\underline{\operatorname{Hom}}_{\Omega_{X}^{*}}\left(\Omega_{X}^{*}\langle Y\rangle, \underline{\operatorname{Hom}}_{X}\left(\Omega_{X}^{*}, K_{X}^{*}\right)\right)
$$

where we have morphisms of $\Omega_{X}^{*}$ modules. We get an isomorphism

$$
\theta: D_{\Omega^{*}}^{\cdot}\langle X-Y\rangle \stackrel{\sim}{\rightarrow} D^{*}\langle X-Y\rangle
$$


by composing with the projection $\operatorname{Hom}_{X}\left(\Omega_{X}^{*}, K_{X}^{*}\right) \rightarrow K_{X}^{*}$ dual to the inclusion $\hat{c}_{X}=\Omega_{X}^{0} \subset \Omega_{X}^{*}$. To see the isomorphism we apply the following rule.

Let $A$ be a ring, $B$ an $A$-algebra, $M$ a $B$-module and $N$ an $A$-module. Then

$$
\operatorname{Hom}_{B}\left(M, \operatorname{Hom}_{A}(B, N)\right) \simeq \operatorname{Hom}_{A}(M, N) .
$$

Let $S K_{X}$ denote the total complex $\underline{\operatorname{Hom}}_{X}\left(\Omega_{X}^{*}, K_{X}^{*}\right)$, with differential $\Delta_{S K_{\dot{X}}}$. Let $d_{X-Y}$ be the differential of $\Omega_{X}^{*}\langle Y\rangle$, and $\Delta_{X-Y}^{\Omega^{*}}$ the differential of $D_{\Omega^{*}}^{*}\langle X-Y\rangle$. Then

$$
\forall \varphi \in \Gamma D_{\Omega^{*}}^{k}\langle X-Y\rangle, \quad \Delta_{X-Y}^{\Omega^{*}}(\varphi)=\Delta_{S K_{X}} \circ \varphi+(-1)^{k+1} \varphi \circ d_{X-Y} .
$$

The differential $\Delta_{X-Y}$ :

Lemma II.1.2. For any $\Psi \in \Gamma D^{*}\langle X-Y\rangle$, let $\Psi_{-1}$ be the composed morphism of $\theta^{-1}(\Psi)$ with the projection $\operatorname{Hom}_{X}\left(\Omega_{X}^{*}, K_{X}^{*}\right) \rightarrow \operatorname{Hom}_{X}\left(\Omega_{X}^{1}, K_{X}^{*}\right)$, dual to the inclusion $\Omega_{X}^{1} \subset \Omega_{X}^{*}$. Write $\Delta_{S K_{X}^{\prime}}=d_{S K_{X}^{\prime}}^{\prime}+(-1)^{i+1} \delta_{S K_{X}^{\prime}}^{\prime}[\mathbf{E}]$. Then $\forall \Psi \in \Gamma D^{k}\langle X-Y\rangle$,

$$
\Delta_{X-Y}(\Psi)=d_{S K_{X}}^{\prime} \circ \Psi_{-1}+(-1)^{*+1} \delta_{K_{X}^{*}} \circ \Psi+(-1)^{k+1} \Psi \circ d_{X-Y} .
$$

Proof. Apply the isomorphism $\theta$ to get $\Delta_{X-Y}$ from $\Delta_{X-Y}^{\Omega^{*}}$.

The element $\Psi_{-1}$ operates as follows:

$$
\begin{aligned}
& \Omega_{X}^{i}\langle Y\rangle \stackrel{\Psi_{-1}}{\rightarrow} \underline{\operatorname{Hom}}_{X}\left(\Omega_{X}^{1}, K_{X}^{1+i+k}\right), \\
& \eta \rightarrow \Psi_{-1}(\eta): \omega \rightarrow(-1)^{k} \Psi(\omega \cdot \eta) .
\end{aligned}
$$

3. The filtrations. Consider the following filtrations on $K_{X}$. (The choice will be explained later.) $W_{i}\left(K_{X}^{\dot{*}}\right)=0$ for $i<2 n+2$, and $W_{i}\left(K_{X}^{\cdot}\right)=K_{X}^{*}$ for $i \geqslant 2 n+2$; $F^{i} K_{\dot{X}}=K_{\dot{X}}$ for $i \leqslant n+1$ and $F^{i} K_{\dot{X}}=0$ for $i \geqslant n+2$. Then we have the dual filtrations on $D^{*}\langle X-Y\rangle$ :

$$
\begin{gathered}
F^{h} D^{\cdot}\langle X-Y\rangle \simeq \underline{\operatorname{Hom}}_{X}\left(\left(\Omega_{X}^{*}\langle Y\rangle / F^{n+2-h}\left(\Omega_{X}^{*}\langle Y\rangle\right), K_{X}^{*}\right),\right. \\
W_{-r+2 n+2} D^{\cdot}\langle X-Y\rangle \simeq \underline{\operatorname{Hom}}_{X}\left(\Omega_{X}^{*}\langle Y\rangle / W_{r-1}\left(\Omega_{X}^{*}\langle Y\rangle\right), K_{X}^{*}\right), \\
\mathrm{Gr}_{-r+2 n+2}^{W} D^{*}\langle X-Y\rangle \simeq \underline{\operatorname{Hom}}_{X}\left(\operatorname{Gr}_{r}^{W}\left(\Omega_{X}^{*}\langle Y\rangle\right), K_{X}^{*}\right) .
\end{gathered}
$$

Definition II.1.3. The complex $D^{*}\langle X-Y\rangle=\operatorname{Hom}_{X}\left(\Omega_{X}^{*}\langle Y\rangle, K_{X}^{*}\right)$ with the differential $\Delta_{X-Y}$ and the dual filtrations defined above, is the dual logarithmic complex in $D^{+} F_{2}(X, \mathbf{C})$.

2. The quasi-isomorphism.

1. We want to define a morphism:

$$
\varphi: \Omega_{X-Y^{(\cdot)}}^{*} \rightarrow D^{*}\langle X-Y\rangle[-2 n-2,-2 n-2] .
$$

With the notations of $I .1$ and I.2 for a N.C.D. $Y$ in $X$, let $y_{j_{0} \cdots j_{m}}$ be the generic point of $Y_{j_{0}} \cap \cdots \cap Y_{j_{m}}$ and $y_{-1}$ for $X$. We define

$$
\varphi_{m}^{\lambda}: \Omega_{Y_{t_{0}} \cap \cdots \cap Y_{I_{m}}}^{\lambda} \rightarrow \underline{\operatorname{Hom}}_{X}\left(\Omega_{X}^{n+1-\lambda}\langle Y\rangle, H_{y_{j_{0} \cdots j_{m}}^{m+1}}\left(\Omega_{X}^{n+1}\right)\right)
$$

and

$$
\varphi_{-1}^{\lambda}: \Omega_{X}^{\lambda} \rightarrow \underline{\operatorname{Hom}}_{X}\left(\Omega_{X}^{n+1-\lambda}\langle Y\rangle, H_{y_{-1}}^{0}\left(\Omega_{X}^{n+1}\right)\right)
$$

as follows. 
Let $\alpha$ be a section of $\Omega_{Y_{j 0} \cap \cdots \cap Y_{j_{m}}}^{\lambda}$, and $\tilde{\alpha}$ a lifting of $\alpha$ in $\Omega_{X, y_{j 0} \ldots j_{m}}^{\lambda}$. Using Grothendieck symbols to denote elements of $K_{X}$, then $\varphi_{m}^{\lambda}(\alpha)$ is obtained (up to a sign) by the compositions of morphisms

$$
\Omega_{X}^{n+1-\lambda}\langle Y\rangle \stackrel{y_{j_{0}} \times \cdots \times y_{j_{m}}}{\rightarrow} \Omega_{X, y_{j_{0} \ldots j_{m}}^{n+1-\lambda}} \stackrel{\left[y_{j_{j}} \cdots y_{j_{m}}\right]}{\rightarrow} H_{y_{j_{0} \ldots j_{m}}^{m+1}}^{y^{\prime}}\left(\Omega_{X}^{n+1}\right)
$$

and $\varphi_{m}^{\lambda}(\alpha)$ will be trivial on $\Omega_{X}^{i}\langle Y\rangle$ for $i \neq n+1-\lambda$.

THEOREM II.2.1. The morphism

$$
\varphi: \Omega_{X-Y^{(\cdot)}}^{*} \underset{\rightarrow}{\rightarrow} D^{\cdot}\langle X-Y\rangle[-2 n-2,-2 n-2]
$$

defined above, is a bifiltered quasi-isomorphism.

Proof. In fact, one should expect this formula up to a sign, since differentials in either side have been chosen independently.

(1) We see by the definition, that $\varphi_{m}^{\lambda}(\alpha)$ of degree $m+\lambda+1$ is trivial on the forms in $\Omega_{X}^{n+1-\lambda}\langle Y\rangle$ not containing $d y_{j_{0}}\left|y_{j_{0}} \wedge \cdots \wedge d y_{j_{m}}\right| y_{j_{m}}$. In particular $\varphi_{m}^{\lambda}(\alpha)$ is trivial on $W_{m} \Omega_{X}^{*}\langle Y\rangle$, so we have

$$
\varphi\left(W_{-m-1} \Omega_{X-Y^{(\cdot)}}^{*}\right) \subset W_{-m-1} D^{*}\langle X-Y\rangle[-2 n-2,-2 n-2] .
$$

Also

$$
\varphi F^{m} \Omega_{X-Y^{(\cdot)}}^{*} \subset F^{m} D^{\cdot}\langle X-Y\rangle[-2 n-2,-2 n-2]
$$

since $\varphi\left(\Omega_{X-Y^{(\cdot)}}^{\lambda}\right)$ is trivial except on $\Omega_{X}^{n+1-\lambda}\langle Y\rangle$, which means $\varphi\left(F^{m}\left(\Omega_{\left.\left.X-Y^{(\cdot)}\right)\right)}^{*}\right.\right.$ is trivial on $F^{n-m+2}\left(\Omega_{X}^{*}\langle Y\rangle\right)$.

2. Compatibility of differentials. We have to compute the three terms of the formula (II.1.2).

$$
\begin{aligned}
\Delta_{X-Y}\left(\varphi_{m}^{\lambda}(\alpha)\right)= & \underbrace{(-1)^{m+\lambda} \varphi_{m}^{\lambda}(\alpha) \circ d_{X-Y}}_{(\mathrm{I})}+\underbrace{d_{S K \dot{x}_{\bar{x}}}^{\prime} \circ\left(\varphi_{m}^{\lambda}(\alpha)\right)_{-1}}_{(\mathrm{II})} \\
& +\underbrace{(-1)^{n+m} \delta_{\bar{x}^{n+n}} \varphi_{m}^{\lambda}(\alpha)}_{(\mathrm{III})} .
\end{aligned}
$$

The term (II). We have

$$
\begin{array}{ccccc}
\Omega_{X}^{n-\lambda}\langle X\rangle & \rightarrow & \underline{\operatorname{Hom}}_{X}\left(\Omega_{X}^{1}, H_{y_{j_{0} \ldots j_{m}}+1}^{m+1}\left(\Omega_{X}^{n+1}\right)\right) \simeq H_{y_{j_{0} \ldots j_{m}}^{m+1}}\left(\Omega_{X}^{n}\right) \rightarrow H_{y_{j_{0} \ldots j_{m}}^{m+1}}^{m}\left(\Omega_{X}^{n+1}\right), \\
\eta & \mapsto & \eta_{-1}=\left(\varphi_{m}^{\lambda}(\alpha)\right)_{-1}(\eta) & \mapsto & d_{S K_{x}}^{\prime} \eta_{-1} .
\end{array}
$$

Let $\eta=\theta \wedge d y_{l_{1}}\left|y_{l_{1}} \wedge \cdots \wedge d y_{l_{\tau}}\right| y_{l_{\tau}}$ with $\theta \in \Gamma \Omega_{X}^{*}$. We have

$$
\eta_{-1}=(-1)^{m+1} \frac{y_{j_{0}} \times \cdots \times y_{j_{m}}}{y_{l_{1}} \times \cdots \times y_{l_{\tau}}}\left[\begin{array}{c}
\tilde{\boldsymbol{\alpha}} \wedge \boldsymbol{\theta} \wedge d y_{l_{1}} \wedge \cdots \wedge d y_{l_{\tau}} \\
y_{j_{0}} \cdots y_{j_{m}}
\end{array}\right]
$$

and

$$
d_{S K_{x} \eta_{-1}}^{\prime}=(-1)^{m+1} \frac{y_{j_{0}} \times \cdots \times y_{j_{m}}}{y_{l_{1}} \times \cdots \times y_{l_{\tau}}}\left[\begin{array}{c}
d(\tilde{\alpha} \wedge \boldsymbol{\theta}) \wedge d y_{l_{l}} \wedge \cdots \wedge d y_{l_{\tau}} \\
y_{j_{0}} \cdots y_{j_{m_{m}}}
\end{array}\right] .
$$


The term (I). We have

$$
(-1)^{m+\lambda} \varphi_{m}^{\lambda}(\alpha) \cdot d_{X-Y} \eta=(-1)^{m+\lambda}\left[\begin{array}{c}
\tilde{\alpha} \wedge d \theta \wedge d y_{l_{1}} \wedge \cdots \wedge d y_{l_{\tau}} \\
y_{j_{0}} \cdots y_{j_{m}}
\end{array}\right] \times \frac{y_{j_{0}} \times \cdots \times y_{j_{m}}}{y_{l_{1}} \times \cdots \times y_{l_{\tau}}} .
$$

Then we compare terms (I) + (II) to the term

$$
\varphi_{m}^{\lambda+1}(d \alpha)(\eta)=\frac{y_{j_{0}} \times \cdots \times y_{j_{m}}}{y_{l_{1}} \times \cdots \times y_{l_{\tau}}}\left[\begin{array}{c}
d \tilde{\alpha} \wedge \boldsymbol{\theta} \wedge d y_{l_{1}} \wedge \cdots \wedge d y_{l_{\tau}} \\
y_{j_{0}} \cdots y_{j_{m}}
\end{array}\right] .
$$

Using the equality $(-1)^{m+1} d(\tilde{\alpha} \wedge \theta)+(-1)^{m+\lambda+1} \tilde{\alpha} \wedge d \theta=(-1)^{m+1} d \tilde{\alpha} \wedge \theta$ we get the equality (I) + (II) $+\left(\right.$ IV), if we admit as a differential $(-1)^{m+1} d \alpha$ in $\Omega_{Y_{j_{0}} \cap \cdots \cap Y_{j_{m}}}^{\lambda}$, which happens naturally when we translate degrees by $m+1$.

The differential $\delta$. The term (III) is written explicitly in the following way:

$$
\begin{aligned}
& \Omega_{X}^{n+1-\lambda}\langle Y\rangle \underset{\varphi_{m}^{\lambda}(\alpha)}{\rightarrow} H_{Y_{t^{0} \cdots j_{m}}^{m+1}}^{m+1}\left(\Omega_{X}^{n+1}\right) \underset{\partial}{\rightarrow} \sum_{t \neq j_{0} \ldots \ldots, j_{m}} H_{Y_{j_{0} \cdots j_{m}}}^{m+2}\left(\Omega_{X}^{n+1}\right), \\
& \frac{\theta \wedge d y_{j_{0}} \wedge \cdots \wedge d y_{j_{m}} \wedge d y_{l_{1}} \wedge \cdots \wedge d y_{l_{\tau}}}{y_{j_{0}} \times \cdots \times y_{j_{m}} \times y_{l_{1}} \times \cdots \times y_{l_{\tau}}} \\
& \rightarrow \sum_{i \in[1, \tau]}\left[\begin{array}{c}
\tilde{\alpha} \wedge \theta \wedge d y_{j_{0}} \wedge \cdots \wedge d y_{j_{m}} \wedge d y_{l_{1}}\left|y_{l_{1}} \wedge \cdots \wedge d y_{l_{i}} \wedge \cdots \wedge d y_{l_{\tau}}\right| y_{l_{\tau}} \\
y_{j_{0}} \cdots y_{j_{m}} y_{l_{i}}
\end{array}\right] .
\end{aligned}
$$

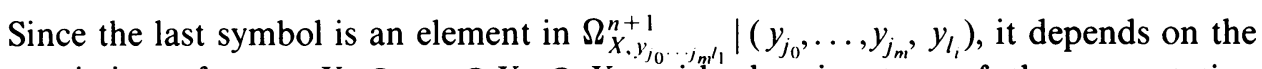
restriction of $\alpha$ to $Y_{j_{0}} \cap \cdots \cap Y_{j_{m}} \cap Y_{l_{l}}$, with the signature of the permutation putting $\left(y_{j_{0}}, \ldots, y_{j_{m}}, y_{l_{1}}\right)$ in an increasing sequence.

Now, the differential in $X-Y^{(\cdot)}$ gives the restriction of $\alpha$ to $Y_{j_{0}} \cap \cdots \cap Y_{j_{m}} \cap Y_{l_{i}}$, with a sign depending on the corresponding permutation of $\left(Y_{j_{0}}, \ldots, Y_{j_{m}}, Y_{l_{i}}\right)$. So we deduce the compatibility of differentials (up to a sign \pm 1 ).

3. Comparision of $\mathrm{Gr}_{*}^{W}$. We have

$$
\begin{gathered}
\mathrm{Gr}_{-q}^{W} \Omega_{X-Y^{(\cdot)}}^{*} \simeq \mathrm{Gr}_{-q+1}^{W} \Omega_{Y^{(\cdot)}}^{*}[-1] \simeq \Omega_{Y^{(q-1)}}^{*}[-q], \\
\mathrm{Gr}_{-q}^{W} D^{\cdot}\langle X-Y\rangle[-2 n-2,-2 n-2] \simeq \mathrm{Gr}_{-q+2 n+2}^{W} D^{\cdot}\langle X-Y\rangle[-2 n-2] \\
\simeq \underline{\operatorname{Hom}}_{X}\left(\mathrm{Gr}_{q}^{W} \Omega_{X}^{*}\langle Y\rangle, K_{X}\right)[-2 n-2] \simeq \underline{\operatorname{Hom}}_{X}\left(\Omega_{Y^{(q-1)}}^{*}[-q], K_{X}^{*}\right)[-2 n-2] \\
\simeq \underline{\operatorname{Hom}}_{Y^{(q-1)}}\left(\Omega_{Y^{(4-1)}}^{*}, K_{Y^{(q-1)}}^{*}\right)[-2 n-2+q] .
\end{gathered}
$$

Then

$$
\mathrm{Gr}_{-q}^{W}(\varphi): \Omega_{Y^{(4-1)}}^{*}[-q] \stackrel{\approx}{\rightarrow} \underline{\operatorname{Hom}}_{Y^{(4-1)}}\left(\Omega_{Y^{(4}}^{*}{ }^{11}, K_{Y^{(4-1)}}^{*}\right)[-2 n-2+q]
$$

is a quasi-isomorphism inducing for each degree $i \in \mathbf{N}$, the duality isomorphism

$$
\Omega_{Y^{(4-1)}}^{i} \simeq \underline{\operatorname{Hom}}_{Y^{(q-1)}}\left(\Omega_{Y^{(q-1)}}^{n+1-q-i}, \Omega_{Y^{(4-1)}}^{n+1-q}\right) .
$$

With our convention the filtration $F$ of $K_{Y^{(q-1)}}$ is given by

$$
F^{n+1-q} K_{Y^{(4-1)}}=K_{Y^{(4-1)}}^{\cdot} \text { and } F^{n+2-q}=0 .
$$


We deduce a filtration $F$ on $\operatorname{Hom}_{Y^{(q-1)}}\left(\Omega_{Y^{(q-1)}}^{*}, K_{Y^{(q-1)}}^{*}\right)[-2 n-2+q]$, and we can check that $\mathrm{Gr}_{-q}^{W}(\varphi)$ respects filtrations $F$, and we have

$$
\mathrm{Gr}_{F}^{\lambda} \mathrm{Gr}_{-q}^{W}(\varphi): \Omega_{Y^{(q-1)}}^{\lambda} \simeq \underline{\operatorname{Hom}}_{Y^{(q-1)}}\left(\Omega_{Y^{(q-1)}}^{n-q^{-\lambda}}, K_{Y^{(q-1)}}^{\cdot}\right)[-n-1+q] .
$$

Then $\mathrm{Gr}_{-q}^{W}(\varphi)$ is a filtered quasi-isomorphism.

COROLlaRY II.2.2. For each $i \in \mathbf{N}$, we have a filtered quasi-isomorphism for induced $W$ :

$$
\operatorname{Gr}_{F}^{i}(\varphi): \Omega_{X-Y^{(\cdot)}}^{i}[-i] \stackrel{\approx}{\rightarrow} \underline{\operatorname{Hom}}_{X}\left(\Omega_{X}^{n+1-i}\langle Y\rangle, K_{X}^{\cdot}\right)[-n-1-i] .
$$

Summary. We state the preceding result, giving duality with value in $K_{X}^{*}$, $\operatorname{Hom}_{X}\left(\Omega_{X}^{*}, K_{X}^{*}\right)$ or $\mathbf{C}$, as a pairing

$$
\begin{array}{ll}
\Omega_{X-Y^{(\cdot)}}^{*} \otimes_{\mathcal{O}_{X}} \Omega_{X}^{*}\langle Y\rangle & \rightarrow K_{X}^{\cdot} \stackrel{\operatorname{Tr}}{\rightarrow} \mathbf{C}, \\
\alpha \otimes \omega & \rightarrow \varphi(\alpha)(\omega), \\
\Omega_{X-Y^{(\cdot)}}^{*} \otimes_{\Omega_{X}^{*}} \Omega_{X}^{*}\langle Y\rangle & \rightarrow \quad \underline{\operatorname{Hom}}_{X}\left(\Omega_{X}^{*}, K_{X}^{*}\right) \stackrel{\operatorname{Tr}}{\rightarrow} \mathbf{C},
\end{array}
$$

corresponding to bifiltered quasi-isomorphisms:

$$
\varphi: \Omega_{X-Y^{(\cdot)}}^{*} \stackrel{\approx}{\rightarrow} D^{\cdot}\langle X-Y\rangle[-2 n-2,-2 n-2]
$$

and

$$
{ }^{t} \varphi: \Omega_{X}^{*}\langle Y\rangle \stackrel{\approx}{\rightarrow} \underline{\operatorname{Hom}}_{X}\left(\Omega_{X-Y^{(\cdot)}}^{*}, K_{X}^{*}\right)[-2 n-2,-2 n-2]
$$

3. Homology. We consider here the homology with no condition on supports, or Borel-Moore homology.

1. Consider the following isomorphisms for $X$ projective and smooth of $\operatorname{dim} n+1$ :

$$
H^{i}(X, \mathbf{C}) \stackrel{P}{\rightarrow} H_{2 n+2-i}(X, \mathbf{C}) \stackrel{D}{\rightarrow} H^{2 n+2-i}(X, \mathbf{C})^{*}
$$

where $P$ stands for Poincaré isomorphism, $D$ for duality and $D \circ P$ for Poincaré duality.

Lemma II.3.1. The Poincaré duality is of type $(-n-1,-n-1)$ on M.H.S.

Proof. On the level of differential forms $D \circ P$ acts as follows:

$$
w \in \Gamma \Omega^{p, q} \rightarrow D \circ P(w): w^{\prime} \in \Gamma \Omega^{n+1-p, n+1-q} \rightarrow \int_{X} w \wedge w^{\prime}
$$

and so $D \circ P(w)$ is of type $(p-n-1, q-n-1)$ in the dual M.H.S. to $H^{2 n+2-i}(X, \mathrm{C})$.

\section{Dual M.H.C.}

Definition II.3.2. (i) Let $Y$ be a N.C.D. in $X$. Consider the following filtrations $W$ and $F$ on the dualizing complex $K_{X}^{*}: W_{i} K_{X}^{*}=K_{X}$ for $i \geqslant 0$ and $W_{i}=0$ for $i<0$, 
$F^{i} K_{X}^{\cdot}=K_{X}$ for $i \leqslant 0$ and $F^{1}=0$ for $i \geqslant 1$. We define the following complexes, with their dual filtrations

$$
\begin{array}{ll}
D_{X-Y}=\underline{\operatorname{Hom}}_{X}\left(\Omega_{\left.X-Y^{(\cdot)}, K_{X}^{*}\right),}^{D_{X}}=\underline{\operatorname{Hom}}_{X}\left(\Omega_{X}^{*}, K_{X}^{*}\right),\right. \\
D_{X-Y}^{c}=\underline{\operatorname{Hom}}_{X}\left(\Omega_{X}^{*}\langle Y\rangle, K_{X}^{*}\right), & D_{Y}=\underline{\operatorname{Hom}}_{X}\left(\Omega_{Y^{(\bullet)}}^{*}, K_{X}^{*}\right)
\end{array} .
$$

(ii) We define the M.H.S. on the homology as follows:

$$
\begin{array}{ll}
H_{i}(X-Y, \mathbf{C})=R^{-i} \Gamma D_{X_{l} Y}, & H_{i}(X, \mathbf{C})=R^{-i} \Gamma D_{X}, \\
H_{i}^{c}(X-Y, \mathbf{C})=R^{-i} \Gamma D_{X-Y}^{c}, & H_{i}(Y, \mathbf{C})=R^{-i} \Gamma D_{Y} .
\end{array}
$$

In fact we have considered in II.2, the duality

$$
\Omega_{X-Y^{(\cdot)}}^{*} \otimes \Omega_{X}^{*}\langle Y\rangle \rightarrow K_{X}^{*}
$$

which gives us

$$
{ }^{t} \varphi: \Omega_{X}^{*}\langle Y\rangle \stackrel{\approx}{\rightarrow} \operatorname{Hom}\left(\Omega_{X-Y^{(\cdot)}}^{*} K_{X}^{*}\right)[-2 n-2,-2 n-2] .
$$

Our new conventions on the filtrations of $K_{X}$ have the effect of lowering the degrees of $W$ by $2 n+2$ and $F$ by $n+1$, so as to get ${ }^{t} \varphi$ of type $(-n-1,-n-1)$, denoted by

$$
\left({ }^{t} \varphi\right)^{\prime}: \Omega_{X}^{*}\langle Y\rangle \stackrel{\approx}{\rightarrow} D_{X-Y}[-2 n-2,-2 n-2] .
$$

In the same way, we have a natural pairing

$$
\Omega_{Y^{(\cdot)}}^{*} \otimes_{\mathfrak{C}_{X}} \Omega_{X}^{*}\langle Y\rangle / \Omega_{X}^{*} \rightarrow K_{X}^{*}
$$

giving rise to the quasi-isomorphism of type $(-n-1,-n-1)$ :

$$
\left({ }^{t} \Psi\right)^{\prime}: \Omega_{X}^{*}\langle Y\rangle / \Omega_{X}^{*} \underset{\rightarrow}{\approx} D_{Y}[-2 n-1,-2 n-1] .
$$

We have the following sequence of quasi-isomorphisms:

$$
\begin{aligned}
& 0 \rightarrow \Omega_{Y^{(\cdot)}}^{*}[-1,-1] \quad \rightarrow \Omega_{X-Y^{(\cdot)}}^{*} \\
& 1 \downarrow \Psi^{\prime} \quad \| \varphi^{\prime} \\
& 0 \rightarrow \underline{\operatorname{Hom}}_{X}\left(\Omega_{X}^{*}\langle Y\rangle / \Omega_{X}^{*}, K_{X}^{*}\right)[-2 n-2,-2 n-2] \rightarrow D_{X-Y}^{c}[-2 n-2,-2 n-2] \\
& \rightarrow \Omega_{X}^{*} \quad \rightarrow 0 \\
& \downarrow C_{X} \\
& \rightarrow D_{X}[-2 n-2,-2 n-2] \rightarrow 0
\end{aligned}
$$

dual to $0 \rightarrow \Omega_{X}^{*} \rightarrow \Omega_{X}^{*}\langle Y\rangle \rightarrow \Omega_{X}^{*}\langle Y\rangle / \Omega_{X}^{*} \rightarrow 0$ and which corresponds to the diagram of cohomology groups with coefficients $C$ :

$$
\begin{array}{ccccccc}
\rightarrow & H_{c}^{i}(X-Y) & \rightarrow & H^{i}(X) & \rightarrow & H^{i}(Y) & \rightarrow \\
& \Downarrow \varphi^{\prime} & & \downarrow C_{X} & & l \downarrow \Psi^{\prime} & \\
\rightarrow \quad H_{2 n+2-i}^{c}(X-Y) & \rightarrow & H_{2 n+2-i}(X) & \rightarrow & \left(H^{2 n+2-i}(Y)\right)^{*} & \rightarrow
\end{array}
$$


3. Duality at spectral sequence level.

COROLlary II.3.3. (i) The spectral sequence ${ }_{W} E_{1}\left(D_{X-Y}\right)$ is the dual with value in $\mathbf{C}$

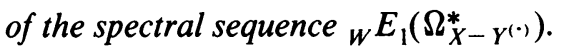

(ii) The Poincaré isomorphism $H^{i}(X-Y, \mathrm{C}) \rightarrow H_{2 n+2-i}(X-Y, \mathrm{C})$ induces from $\mathrm{Gr}_{q}^{W}$ to $\mathrm{Gr}_{q-n-1}^{W}$, the Poincaré isomorphism on $Y^{(q-i-1)}$.

The duality is given in this way: to an element $\eta \in H_{i}(X-Y)=R^{-i} \Gamma D_{X-Y}$

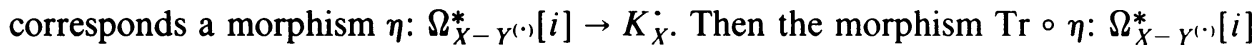
$\rightarrow K_{X} \rightarrow \mathrm{C}$ induces a morphism $H_{c}^{i}(X-Y) \rightarrow \mathrm{C}$.

4. Duality in $D^{+} F(X, \mathbf{Q})$.

Proposition II.4.1. Suppose $W_{-1} \mathbf{Q}_{X}=0$ and $W_{0} \mathbf{Q}_{X}=\mathbf{Q}_{X}$, then there exists $a$ filtered isomorphism in $D^{+} F(X, \mathbf{Q})$ :

$$
{ }^{t} \varphi^{\mathbf{Q}}:\left(\mathbf{R} j_{*} \mathbf{Q}_{X-Y}, \tau\right) \rightarrow \mathbf{R} \underline{\operatorname{Hom}} \mathbf{Q}\left(\mathbf{Q}_{X-Y^{(\cdot)}}, \mathbf{Q}_{X}\right)
$$

which fits in a commutative diagram in $D^{+} F(X, \mathrm{C})$ :

$$
\begin{aligned}
\left(\mathbf{R} j_{*} \mathbf{Q}_{X-Y}, \tau\right) \otimes \mathbf{C} & \stackrel{\sim}{\rightarrow} \mathbf{R} \operatorname{Hom}_{\mathbf{Q}}\left(\mathbf{Q}_{X-Y^{(\cdot)}}, \mathbf{Q}_{X}\right) \otimes \mathbf{C} \\
\downarrow \downarrow & \stackrel{\imath}{ } \\
\Omega_{X}^{*}\langle Y\rangle & \stackrel{\mathbf{R}}{\text { Hom }_{X}}\left(\Omega_{X-Y^{(\cdot)}}^{*}, K_{X}^{*}\right)[-2 n-2,-2 n-2]
\end{aligned}
$$

Proof. The exact sequence $0 \rightarrow j ! \mathbf{Q}_{X-Y} \rightarrow \mathbf{Q}_{X} \rightarrow i_{*} \mathbf{Q}_{Y} \rightarrow 0$ gives a quasi-isomorphism $j ! \mathbf{Q}_{X-Y} \underset{\sim}{\stackrel{\approx}{\rightarrow}} \mathbf{Q}_{X-Y^{(\bullet)}}$. Its dual with value in an injective resolution $I_{X}$ of $\mathbf{Q}_{X}$

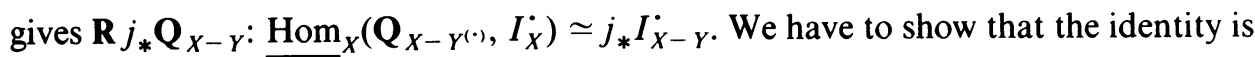

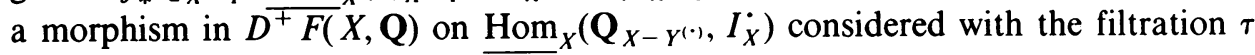
and the filtration $W$.

$$
\begin{array}{cccccc}
I_{X}^{0} & \rightarrow & I_{X}^{1} & \rightarrow & I_{X}^{2} & \rightarrow \\
\uparrow & & \uparrow & & \uparrow & \\
\operatorname{Hom}\left(\mathbf{Q}_{Y^{(0)}}, I_{X}^{0}\right) & \rightarrow & \operatorname{Hom}\left(\mathbf{Q}_{Y^{(0)}}, I_{X}^{1}\right) & \rightarrow & \operatorname{Hom}\left(\mathbf{Q}_{Y^{(0)}}, I_{X}^{2}\right) & \rightarrow \\
\uparrow & & \uparrow & & \uparrow & \\
\operatorname{Hom}\left(\mathbf{Q}_{Y^{(1)}}, I_{X}^{0}\right) & \rightarrow & \operatorname{Hom}\left(\mathbf{Q}_{Y^{(1)}}, I_{X}^{1}\right) & \rightarrow & \operatorname{Hom}\left(\mathbf{Q}_{Y^{(1)}}, I_{X}^{2}\right) & \rightarrow \\
\uparrow & & \uparrow & & \uparrow
\end{array}
$$

We see on this figure that the two filtrations are incomparable, but we can use the following fact.

Let $\Pi: Y^{(m)} \rightarrow Y$ be the finite projection then we have in

$$
D^{+}(X, \mathbf{Q}): \Pi_{*} \mathbf{Q}_{Y^{(m)}}[-2 m-2] \simeq \mathbf{R} \operatorname{Hom}\left(\Pi_{*} \mathbf{Q}_{Y^{(m)}}, \mathbf{Q}_{X}\right) .
$$

We now construct a new complex $D^{\mathbf{Q}}$ quasi-isomorphic to $\operatorname{Hom}\left(\mathbf{Q}_{X-Y^{(\cdot)}, I_{X}}\right)$. For any variety $T$, let $D_{T}^{*}$ be the dualizing complex with coefficients $\mathbf{Q}$ in degrees 
[-2 $\operatorname{dim} T, 0]$. Define $D^{\mathbf{Q}}$ as the simple complex associated to

$$
\begin{aligned}
& D_{X}^{-2 n-2} \rightarrow D_{X}^{-2 n-1} \rightarrow \begin{array}{c}
D_{X}^{-2 n} \\
\uparrow
\end{array} \quad \rightarrow \quad D_{X}^{-2 n+1} \rightarrow \begin{array}{c}
D_{X}^{-2 n+2} \\
\uparrow
\end{array} \rightarrow \\
& 0 \quad 0 \quad \rightarrow \quad \Pi_{*} D_{Y^{(0)}}^{-2 n} \rightarrow \Pi_{*} D_{Y^{(0)}}^{-2 n+1} \rightarrow \Pi_{*} D_{Y^{(0)}}^{-2 n+2} \rightarrow \\
& \begin{array}{llllll}
0 & 0 & \rightarrow & 0 & \rightarrow & \Pi_{*} D_{Y^{(1)}}^{-2 n+2}
\end{array} \rightarrow \\
& \begin{array}{llllll}
0 & 0 & 0 & \rightarrow & 0 & 0
\end{array} \\
& \uparrow
\end{aligned}
$$

Then the filtration $\tau\left(D^{\mathbf{Q}}\right)$ is included in $W\left(D^{\mathbf{Q}}\right)$ and the identity is a filtered quasi-isomorphism.

COROLLARY II.4.2. There exists a filtered quasi-isomorphism in $D^{+} F(X, \mathbf{Q})$ :

$$
\Psi^{\mathbf{Q}}:\left(i_{*} \mathbf{Q}_{Y^{(\cdot)}}, W\right) \rightarrow \mathbf{R} \underline{\operatorname{Hom}}_{X}\left(C_{M}\left(\mathbf{Q}_{X} \rightarrow \mathbf{R} j_{*} \mathbf{Q}_{X-Y}\right)[-1,-1], \mathbf{Q}_{X}\right)
$$

which is compatible to $\Psi$ defined with coefficients $\mathbf{C}$.

5. Gysin morphism at the C.M.H.C. level. Let $f: X^{\prime} \rightarrow X$ be a morphism of proper varieties of $\operatorname{dim} n^{\prime}+1$ and $n+1$. Let $Y^{\prime}$ be a N.C.D. in $X^{\prime}$, and $Y$ a N.C.D. in $X$ such that $f^{-1}(Y)=Y^{\prime}$. We have dual diagrams of cohomology groups with coefficients $\mathbf{C}$, and M.H.S.:

$$
\begin{aligned}
& H_{Y^{\prime}}^{l}\left(X^{\prime}\right) \quad \rightarrow \quad H^{\prime}\left(X^{\prime}\right) \quad \rightarrow \quad H^{\prime}\left(X^{\prime}-Y^{\prime}\right) \\
& \uparrow f^{*} \quad \uparrow f^{*} \quad \uparrow f^{*} \\
& H_{Y}^{l}(X) \quad \rightarrow \quad H^{\prime}(X) \rightarrow H^{\prime}(X-Y) \\
& H_{c}^{2 n^{\prime}+2-l}\left(X^{\prime}-Y^{\prime}\right) \quad \rightarrow \quad H^{2 n^{\prime}+2-l}\left(X^{\prime}\right) \quad \rightarrow \quad H^{2 n^{\prime}+2-l}\left(Y^{\prime}\right) \\
& \text { '(D) } \quad \downarrow \operatorname{Tr} f / X-Y \quad \downarrow \operatorname{Tr} f \quad \downarrow \operatorname{Tr} f / Y \\
& H_{c}^{2 n+2-l}(X-Y) \rightarrow H^{2 n+2-l}(X) \rightarrow H^{2 n+2-l}(Y)
\end{aligned}
$$

At the complex level, the Gysin morphism $\operatorname{Tr} f / X-Y$ is defined as dual to $\Omega_{X}^{*}\langle Y\rangle \stackrel{f^{*}}{\rightarrow} \Omega_{X^{\prime}}^{*}\left\langle Y^{\prime}\right\rangle$ :

$$
\begin{aligned}
\Omega_{X^{\prime}-Y^{\prime(\cdot)}}^{*} & \simeq \mathbf{R} \underline{\operatorname{Hom}}_{X^{\prime}}\left(\Omega_{X^{\prime}}^{*}\left\langle Y^{\prime}\right\rangle, K_{X^{\prime}}^{*}\right)\left[-2 n^{\prime}-2,-2 n^{\prime}-2\right] \\
& \stackrel{f^{*}}{\rightarrow} \mathbf{R} \underline{\operatorname{Hom}}_{X}\left(\Omega_{X}^{*}\langle Y\rangle, K_{X}^{*}\right)\left[-2 n^{\prime}-2,-2 n^{\prime}-2\right] \\
& \simeq \Omega_{X-Y^{(\cdot)}\left[-2\left(n^{\prime}-n\right),-2\left(n^{\prime}-n\right)\right] .}
\end{aligned}
$$

The same is true for $\operatorname{Tr} f$ and $\operatorname{Tr} f / Y$, and all can be defined with coefficients in $\mathbf{Q}$.

6. Regular forms on $Y$.

Proposition II.6.1. (i) We define the sheaf of regular forms on a N.C.D. Y in X, of degree $i \in \mathbf{N}$, to be

$$
\tilde{\Omega}_{Y}^{i}=\operatorname{ker}\left(\Omega_{Y^{(0)}}^{i} \rightarrow \Omega_{Y^{(1)}}^{i}\right)
$$


(ii) The complex $\Omega_{Y^{(\cdot)}}^{i}$ is a resolution to the right of $\tilde{\Omega}_{Y}^{i}$, and we have

$$
\begin{aligned}
\tilde{\Omega}_{Y}^{i} & \simeq \operatorname{Ext}_{X}^{1}\left(\Omega_{X}^{n+1-i}\langle Y\rangle / \Omega_{X}^{n+1-i}, \Omega_{X}^{n+1}\right) \\
& \approx \mathbf{R} \underline{\operatorname{Hom}}_{X}\left(\Omega_{X}^{n+1-i}\langle Y\rangle / \Omega_{X}^{n+1-i}, \Omega_{X}^{n+1}\right) .
\end{aligned}
$$

Proof. As in Corollary II.2.2, we have a filtered quasi-isomorphism for induced $W$ :

$$
\mathrm{Gr}_{F}^{i}(\Psi): \Omega_{Y^{(\cdot)}}^{i}[-i] \stackrel{\approx}{\rightarrow} \underline{\operatorname{Hom}}_{X}\left(\Omega_{X}^{n+1-i}\langle Y\rangle / \Omega_{X}^{n+1-i}, K_{X}\right)[-n-i] .
$$

Taking cohomology, we get

$$
\underline{H}^{j}\left(\Omega_{Y^{(\cdot)}}^{i}\right) \simeq \underline{\operatorname{Ext}}_{X}^{j+1}\left(\Omega_{X}^{n+1-i}\langle Y\rangle / \Omega_{X}^{n+1-i}, \Omega_{X}^{n+1}\right) .
$$

It is clear that $\underline{\operatorname{Ext}}_{X}^{0}=0$, and the locally free resolution

$$
\overline{0} \rightarrow \Omega_{X}^{n+1-i} \rightarrow \Omega_{X}^{n+1-i}\langle Y\rangle \rightarrow \Omega_{X}^{n+1-i}\langle Y\rangle / \Omega_{X}^{n+1-i} \rightarrow 0
$$

shows that $\underline{\operatorname{Ext}}_{X}^{j+1}=0$ for $j>0$.

COROLlaRY II.6.2. (i) The complex $\operatorname{Hom}_{X}\left(\Omega_{Y^{(\cdot)}}^{i}, K_{X}^{l}\right)$ is a resolution to the left of $\operatorname{Hom}_{X}\left(\tilde{\Omega}_{Y}^{i}, K_{X}^{l}\right)$ for each $(i, l) \in \mathbf{Z}^{2}$.

(ii) The trace morphism $\operatorname{Tr}: \operatorname{Hom}_{X}\left(\Omega_{Y^{(\cdot)}}^{*}, K_{X}^{*}\right) \rightarrow \operatorname{Hom}_{X}\left(\Omega_{X}^{*}, K_{X}^{*}\right)$ has as image $\operatorname{Hom}_{X}\left(\tilde{\Omega}_{Y}^{*}, K_{X}^{*}\right)$ and the complex $\overline{\operatorname{Hom}}_{X}\left(\Omega_{Y^{(\cdot)}}^{*}, K_{X}^{*}\right)$ is a left resolution of this image respecting the filtration $F$.

(iii) For each $i \in \mathbf{N}$, the complex $\underline{\operatorname{Hom}}_{X}\left(\tilde{\Omega}_{Y}^{i}, K_{X}\right)$ is a resolution of

$$
\Omega_{X}^{n+1-i}\langle Y\rangle / \Omega_{X}^{n+1-i}
$$

embedded in $\underline{\Gamma}_{Y} \underline{\operatorname{Hom}}_{X}\left(\Omega_{X}^{*}, K_{X}^{*}\right)$.

Proof. Consider the exact sequence $\Omega_{X}^{*} \rightarrow \tilde{\Omega}_{Y}^{*} \hookrightarrow \Omega_{Y^{(\cdot)}}^{*}$ and its dual

$$
\underline{\operatorname{Hom}}_{X}\left(\Omega_{Y^{(\cdot)}}^{*} K_{X}^{*}\right) \rightarrow \underline{\operatorname{Hom}}_{X}\left(\tilde{\Omega}_{Y}^{*}, K_{X}^{*}\right) \hookrightarrow \underline{\operatorname{Hom}}_{X}\left(\Omega_{X}^{*}, K_{X}^{*}\right) .
$$

ReMark. Dubois [D] has shown that the C.M.H.C. defined in [H III] are unique up to quasi-isomorphisms respecting the Hodge filtration $F$ only. This leads also to the concept of regular forms on a singular variety.

III. M.H.S. of an embedded variety. Let $X$ be a smooth variety and $i: Y \rightarrow X$ a closed embedding, $j: X-Y \rightarrow X$ the open embedding. We want to construct the M.H.S. of $Y$ using the exact sequence

$$
0 \rightarrow j ! \mathbf{Z}_{X-Y} \rightarrow \mathbf{Z}_{X} \rightarrow i_{*} \mathbf{Z}_{Y} \rightarrow 0
$$

To simplify the proof and notation, we first consider the proper case in detail.

THeOREM III.1.1. With the notation of hypothesis 1 in Part I, let $K_{X}\left(\right.$ resp. $\left.K_{Y^{\prime}}, K_{X^{\prime}}\right)$ denote the C.M.H.C. described in I for the smooth and complete variety $X$ (resp. N.C.D. $Y^{\prime}$ and $X^{\prime}$ ). Then the M.H.S. on the cohomology of the variety $Y$ can be described by the following C.M.H.C. mixed cone:

$$
K_{Y, X}=C_{M}\left(R p_{*} K_{X^{\prime}} \stackrel{i^{\prime *}-\operatorname{Tr} p}{\rightarrow} R p_{*} K_{Y^{\prime}} \oplus K_{X}\right)
$$


The canonical isomorphism $\mathbf{Z}_{Y} \rightarrow R p_{*} K_{Y^{\prime}}^{\mathbf{Z}} \rightarrow K_{Y, X}^{\mathbf{Z}}$ gives a long exact sequence of M.H.S.

$$
0 \rightarrow H^{i}\left(X^{\prime}, C\right) \stackrel{i^{\prime *}-\operatorname{Tr} p}{\rightarrow} H^{i}\left(Y^{\prime}, C\right) \oplus H^{i}(X, C) \stackrel{\operatorname{Tr} p / Y+i^{*}}{\rightarrow} H^{i}(Y, C) \rightarrow 0
$$

which shows that:

(1) $0 \rightarrow H^{i}\left(X^{\prime}, C\right) \rightarrow \mathrm{Gr}_{i}^{W} H^{i}\left(Y^{\prime}, C\right) \oplus H^{i}(X, C) \rightarrow \mathrm{Gr}_{i}^{W} H^{i}(Y, C) \rightarrow 0$ is exact.

(2) $\mathrm{Gr}_{q}^{W} H^{i}(Y, C) \simeq \mathrm{Gr}_{q}^{W} H^{i}\left(Y^{\prime}, C\right)$ for $q<i$.

(3) $\mathrm{Gr}_{q}^{W} H^{i}(Y, C)=0$ for $q>i$ and $q<0$.

I want to state also the following elementary formulation of the theorem.

TheOREM III.1.1'. With the notation of hypothesis 1 in Part I, the canonical M.H.S. on the cohomology of the variety $Y$ is defined by the bifiltered cone in $C^{+} F_{2}(X, \mathbf{C})$ :

$$
K_{Y, X}^{\mathbf{C}}=C_{M}\left(\mathbf{R} p_{*} \Omega_{X^{\prime}}^{*} \stackrel{i^{\prime *}-\operatorname{Tr} p}{\rightarrow} \mathbf{R} p_{*} \Omega_{Y^{\prime} \cdot(\cdot)}^{*} \oplus \mathbf{R} \operatorname{Id}_{*} \Omega_{X}^{*}\right)
$$

To be precise, we mean to choose resolutions representing $R p_{*} \Omega_{X^{\prime}}^{*}, \mathbf{R} p_{*} \Omega_{Y^{(\cdot)}}^{*}$ and $\mathbf{R} \operatorname{Id}_{*} \Omega_{X}^{*}$ such that $i^{*}$ and $\operatorname{Tr} p$ are defined in $C^{+} F_{2}(X, \mathbf{C})$. Then the filtration $W$ induced on $H^{*}(Y, \mathrm{C})$ is defined over $\mathbf{Q}$, so that the filtrations $W$ and $F$ induced on $H^{*}(Y, \mathrm{C})$ give the M.H.S. This construction does not depend on the choices made above, nor on $X$ nor on $X^{\prime}$, and is functorial in $Y$.

Proof. Consider the diagram in $D^{+}(X, \mathbf{Q})$ :

$$
\begin{array}{ccccc}
j ! \mathbf{Q}_{X-Y} & \rightarrow & \mathbf{Q}_{X} & \stackrel{i^{*}}{\rightarrow} & i_{*} \mathbf{Q}_{Y} \\
\operatorname{Tr} p / X-Y \uparrow \lll \downarrow p_{X-Y}^{*} & & \operatorname{Tr} p \uparrow \downarrow p^{*} & & p_{Y}^{*} \downarrow \uparrow \operatorname{Tr} p / Y \\
\mathbf{R} p_{*} j_{!}^{\prime} \mathbf{Q}_{X^{\prime}-Y^{\prime}} & \rightarrow & \mathbf{R} p_{*} \mathbf{Q}_{X^{\prime}} & \stackrel{i^{\prime *}}{\rightarrow} & i_{*} \mathbf{R} p_{Y, *} \mathbf{Q}_{Y^{\prime}}
\end{array}
$$

The lines are triangles, and the composed morphism $\operatorname{Tr} p \circ p^{*}$ is the identity. We deduce easily the following quasi-isomorphism:

$$
i_{*} \mathbf{Q}_{Y} \stackrel{p_{Y}^{*}}{\underset{\sim}{\rightarrow}} C\left(\mathbf{R} p_{*} \mathbf{Q}_{X^{\prime}} \stackrel{i^{\prime *}-\operatorname{Tr} p}{\rightarrow} i_{*} \mathbf{R} p_{Y, *} \mathbf{Q}_{\left.Y^{\prime} \cdot \cdot\right)} \oplus \mathbf{R} \operatorname{Id}_{*} \mathbf{Q}_{X}\right)
$$

We can use, for example, dualizing complexes $D_{X^{\prime}}$ in $\mathrm{Ob} C^{+}\left(X^{\prime}, \mathbf{Q}\right)$ and $D_{X}$ in Ob $C^{+}(X, \mathbf{Q})$ to represent the Gysin morphism in $C^{+}(X, \mathbf{Q})[\mathbf{V}]$ :

$$
\operatorname{Tr} p: p_{*} D_{X^{\prime}}[-2 n-2] \rightarrow D_{X}[-2 n-2], \quad \operatorname{dim} X=n+1,
$$

and Godement resolutions to represent $i^{\prime *}$ in $C^{+}(X, \mathbf{Q})$ :

$$
i^{\prime *}: p_{*} G\left(\mathbf{Q}_{X^{\prime}}\right) \rightarrow i_{*} p_{Y, *} G\left(\mathbf{Q}_{Y^{\prime}}\right) \text {. }
$$

Then we find a complex $K$ with quasi-isomorphisms in $C^{+}(X, \mathbf{Q})$ :

$$
K \stackrel{\approx}{\rightarrow} p_{*} L_{X^{\prime}}[-2 n-2] \text { and } K \underset{\rightarrow}{\approx} p_{*} G\left(\mathbf{Q}_{X^{\prime}}\right)
$$

such that $i^{*}$ and $\operatorname{Tr} p$ are defined together on $K$. 
Define the filtration $W$ as follows:

$$
K_{Y, X}^{\mathbf{Q}}=C_{M}\left(\mathbf{R} p_{*} \mathbf{Q}_{X^{\prime}} \stackrel{i^{\prime *}-\operatorname{Tr} p}{\rightarrow} i_{*} \mathbf{R} p_{Y, *} \mathbf{Q}_{\left.Y^{\prime} \cdot \cdot\right)} \oplus \mathbf{R} \operatorname{Id}_{*} \mathbf{Q}_{X}\right)
$$

The normalisation. If we change the choice of resolutions, we get a commutative diagram in $D^{+}(X, \mathbf{Q})$ :

$$
\begin{aligned}
& p_{Y}^{*}>K_{Y, X}^{\mathbf{Q}} \\
& i_{*} \mathbf{Q}_{Y} \quad I \downarrow \\
& p_{Y}^{*} \supset K_{Y, X}^{\prime \mathbf{Q}}
\end{aligned}
$$

where $K_{Y, X}^{\prime \mathbf{Q}}$ is the new complex, and the isomorphism $I$ in $D^{+} F(X, \mathbf{Q})$ is defined in the Remark 0.2 .5 in a noncanonical way. The diagram is commutative however because $p_{Y}^{*}$ factors through $\mathbf{R} p_{Y, *} \mathbf{Q}_{\left.Y^{\prime} \cdot\right)}$ whose embedding in $K_{Y, X}^{\mathbf{Q}}$ commutes with $I$. We call this fact a normalisation of $K_{Y, X}^{\mathbf{Q}}$. The morphism $(\operatorname{Tr} p) / Y+i_{*}$ is an inverse to $p_{Y}^{*}$ in $D^{+}(X, \mathbf{Q})$.

We define $K_{Y, X}^{\mathrm{C}}$ in the same way, where we use $\operatorname{Hom}\left(\Omega_{X^{\prime}}^{*}, K_{X^{\prime}}^{*}\right)[-2 n-2]$ to define $\operatorname{Tr} p$, since $K_{X^{\prime}}$ is the dualizing complex for $\theta_{X^{\prime}}$-modules.

The filtered isomorphism. We have in $D^{+} F(X, \mathbf{C})$ the canonical isomorphisms

$$
\mathbf{R} p_{*} \mathbf{Q}_{X^{\prime}} \otimes \mathbf{C} \simeq \mathbf{R} p_{*} \Omega_{X^{\prime}}^{*}, \quad i_{*} \mathbf{R} p_{Y, *} \mathbf{Q}_{\left.Y^{\prime} \cdot\right)} \otimes \mathbf{C} \simeq i_{*} \mathbf{R} p_{Y, *} \Omega_{Y^{\prime} \cdot \cdot}^{*}
$$

and $R \operatorname{Id}_{*} \mathbf{Q}_{X} \otimes \mathbf{C} \simeq \mathbf{R} \operatorname{Id}_{*} \Omega_{X}^{*}$. Using Remark 0.2.5, we deduce the following noncanonical isomorphism in $D^{+} F(X, \mathbf{Q})$ :

$$
\left(K_{Y, X}^{\mathbf{Q}}, W\right) \otimes \mathbf{C} \simeq\left(K_{Y, X}^{\mathbf{C}}, W\right) .
$$

But the normalisation property still gives us a commutative diagram in $D^{+}(X, \mathbf{C})$ :

$$
\begin{array}{ccccc}
i_{*} \mathbf{Q}_{Y} \otimes \mathbf{C} & \rightarrow & i_{*} \mathbf{R} p_{Y, *} \mathbf{Q}_{Y^{\prime(\cdot)}} \otimes \mathbf{C} & \rightarrow & K_{Y, X}^{\mathbf{Q}} \otimes \mathbf{C} \\
\downarrow l & & \downarrow l & & \downarrow l \\
i_{*} \mathbf{C}_{Y} & \rightarrow & i_{*} \mathbf{R} p_{Y, *} \Omega_{Y^{\prime} \cdot()}^{*} & \rightarrow & K_{Y, X}^{\mathbf{C}}
\end{array}
$$

In conclusion we have the following lemma.

LemMA III.1.2. (1) With the notation above, we define a C.M.H.C. $K_{Y, X}$ on $X$, as follows:

(i) $i_{*} \mathbf{Z}_{Y}$ in $\mathrm{Ob} D^{+}(X, \mathbf{Z})$.

(ii)

$$
\left(K_{Y, X}^{\mathbf{Q}}, W\right)=C_{M}\left(\mathbf{R} p_{*} \mathbf{Q}_{X^{\prime}} \stackrel{i^{\prime *}-\operatorname{Tr} p}{\rightarrow} i_{*} \mathbf{R} p_{Y, *} \mathbf{Q}_{Y^{\prime \cdot}} \oplus \mathbf{R} \operatorname{Id}_{*} \mathbf{Q}_{X}\right)
$$

in $\mathrm{Ob} D^{+} F(X, \mathbf{Q})$.

(iii) $A$ canonical isomorphism in $D^{+}(X, \mathbf{Q})$ :

$$
\alpha: i_{*} \mathbf{Q}_{Y} \rightarrow i_{*} \mathbf{R} p_{Y, *} \mathbf{Q}_{Y^{(\cdot)}} \rightarrow K_{Y, X}^{\mathbf{Q}} .
$$

(iv)

$$
\left(K_{Y, X}^{\mathbf{C}}, W, F\right)=C_{M}\left(\mathbf{R} p_{*} \Omega_{X^{\prime}}^{*} \stackrel{i^{\prime *}-\operatorname{Tr} p}{\rightarrow} i_{*} \mathbf{R} p_{Y, *} \Omega_{Y^{\prime}}^{*} \oplus \mathbf{R}_{*} \operatorname{Id}_{X}^{*}\right)
$$

in $\mathrm{Ob} D^{+} F_{2}(X, \mathrm{C})$. 
(v) A noncanonical isomorphism in $D^{+} F(X, \mathbf{C})$ :

$$
\left(K_{Y, X}^{\mathbf{Q}}, W\right) \otimes \mathbf{C} \simeq\left(K_{Y, X}^{\mathbf{Q}}, W\right) .
$$

(2) This C.M.H.C. has the following normalisation data:

(i) A canonical isomorphism in $D^{+}(X, \mathbf{C})$ :

$$
p_{Y}^{*}: i_{*} \mathbf{C}_{Y} \rightarrow i_{*} \mathbf{R} p_{Y, *} \Omega_{Y^{\prime(\cdot)}}^{*} \rightarrow K_{Y, X}^{\mathbf{C}} .
$$

(ii) A commutative diagram in $D^{+}(X, \mathbf{C})$ :

$$
\begin{array}{ccc}
i_{*} \mathbf{Q}_{Y} \otimes \mathbf{C} & \stackrel{\sim}{\vec{\alpha}} & K_{Y, X}^{\mathbf{Q}} \otimes \mathbf{C} \\
\imath \downarrow & & \imath \downarrow \\
i_{*} \mathbf{C}_{Y} & \stackrel{p_{Y}^{*}}{\rightarrow} & K_{Y, X}^{\mathbf{C}} \otimes \mathbf{C}
\end{array}
$$

(3) The compatibility property. Let $K_{Y, X}^{\prime}$ be a different C.M.H.C. constructed as above with different choice of resolutions, then there exist isomorphisms

$$
\begin{aligned}
\left(K_{Y, X}^{\mathbf{Q}}, W\right) & \stackrel{\sim}{\rightarrow}\left(K_{Y, X}^{\prime \mathbf{Q}}, W\right) \text { in } D^{+} F(X, \mathbf{Q}) \text { and } \\
\left(K_{Y, X}^{\mathbf{C}}, W, F\right) & \stackrel{\sim}{\rightarrow}\left(K_{Y, X}^{\prime \mathbf{C}}, W, F\right) \text { in } D^{+} F_{2}(X, \mathbf{C})
\end{aligned}
$$

whose restriction to $D^{+}(X, \mathbf{Q})$ and $D^{+}(X, \mathbf{C})$ is compatible with the normalisation data. Furthermore we have a diagram in $D^{+} F(X, \mathbf{C})$ :

$$
\begin{array}{ccc}
\left(K_{Y, X}^{\mathbf{Q}}, W\right) \otimes \mathbf{C} & \stackrel{\sim}{\rightarrow}\left(K_{Y, X}^{\mathbf{C}}, W\right) \\
\downarrow & \downarrow) \\
\left(K_{Y, X}^{\prime \mathbf{Q}}, W\right) \otimes \mathbf{C} & \stackrel{\sim}{\rightarrow}\left(K_{Y, X}^{\prime \mathbf{C}}, W\right)
\end{array}
$$

whose image in $D^{+}(X, \mathbf{C})$ is commutative, since it is compatible with normalisation.

Proof of THEOREM, CONTINUED. Applying the functor of global sections, we find a M.H.C. $\mathbf{R} \Gamma\left(X, K_{Y, X}\right)$ quasi-isomorphic to $\mathbf{R} \Gamma\left(Y, \mathbf{Q}_{Y}\right)$. Now Deligne's fundamental theorem on C.M.H.C. gives us a M.H.S. on $H^{*}(Y)$.

Independence of choice of resolutions. Consider the following diagram:

$$
\begin{array}{ccc}
\left(\mathbf{R} \Gamma\left(X, K_{Y, X}^{\mathbf{Q}}\right), W\right) \otimes \mathbf{C} & \stackrel{\sim}{\rightarrow}\left(\mathbf{R} \Gamma\left(X, K_{Y, X}^{\mathbf{C}}\right), W\right) \\
\downarrow \downarrow & & \downarrow\rangle \\
\left(\mathbf{R} \Gamma\left(X, K_{Y, X}^{\prime \mathbf{Q}}\right), W\right) \otimes \mathbf{C} & \stackrel{\sim}{\rightarrow} & \left(\mathbf{R} \Gamma\left(X, K_{Y, X}^{\prime \mathbf{C}}\right), W\right)
\end{array}
$$

Since this diagram is commutative in $D^{+} \mathbf{C}$ and compatible with normalisation, we have the following commutative diagram on cohomology:

$$
\begin{aligned}
& H^{*}(Y, \mathbf{Q}) \otimes \mathbf{C} \stackrel{\sim}{\rightarrow} H^{*}\left(X, K_{Y, X}^{\mathbf{Q}} \otimes \mathbf{C}\right) \stackrel{\sim}{\rightarrow} H^{*}\left(X, K_{Y, X}^{\mathrm{C}}\right)
\end{aligned}
$$

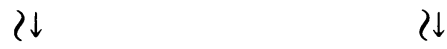

$$
\begin{aligned}
& H^{*}\left(X, K_{Y, X}^{\prime \mathbf{Q}} \otimes \mathbf{C}\right) \stackrel{\sim}{\rightarrow} H^{*}\left(X, K_{Y, X}^{\prime \mathbf{C}}\right)
\end{aligned}
$$


This diagram is also compatible with filtrations since the corresponding isomorphisms used underlie filtered isomorphisms.

REMARK. The "second homotopy" problem prevents us from obtaining the functorial C.M.H.C., however "Normalisation" allows us to obtain the functorial M.H.S. independent of the choice of $i: Y \rightarrow X$ or $p: X^{\prime} \rightarrow X$. We could expect that, since we have shown in Part I that the M.H.S. obtained are those of Deligne. However, in order to be completely independent of [H III], we prove the functoriality in the Appendix.

Remarks. 1. The fact that the long exact sequence splits into short exact sequences has been fundamental in Part I; here it also plays a role in getting the independence of the choices in the construction of the C.H.M.C. In §IV we need to introduce a similar fact.

2. The complex $K_{X, Y}^{\mathbf{C}}$ is a resolution of the sheaf $\mathbf{C}_{Y}$. We may obtain in this way the DeRham cohomology of an algebraic variety, embeddable in a smooth variety.

2. Cohomology with support on $Y$.

Proposition III.2.1. With the notation of Theorem III.1.1, the canonical M.H.S. on $H_{Y}^{*}(X, \mathbf{C})$, the cohomology of $X$ with support on $Y$, is defined by the bifiltered cone

$$
{ }_{h} K_{Y, X}^{\mathbf{C}}=C_{M}\left(\mathbf{R} \operatorname{Id}_{*} \Omega_{X}^{*} \stackrel{j^{\prime *} \circ p^{*}}{\rightarrow} \mathbf{R} p \Omega_{*} \Omega_{X^{\prime}}^{*}\left\langle Y^{\prime}\right\rangle\right) .
$$

Proof. As before, we mean that the filtration $W$ on $H_{Y}^{*}(X, \mathbf{C})$, deduced by means of the spectral sequence, is already defined over $\mathbf{Q}$, and the M.H.S. obtained is independent of any choice. We can prove this again using the triangle

$$
\mathbf{R} \Gamma_{Y}(X, \mathbf{C}) \rightarrow \mathbf{R} \Gamma(X, \mathbf{C}) \rightarrow \mathbf{R} \Gamma(X-Y, \mathbf{C}) .
$$

Proposition III.2.2. The dual complex to $K_{Y, X}$ in Theorem III.1.1

$$
K_{Y, X}^{t}=C_{M}\left(\mathbf{R} p_{*} K_{Y^{\prime}}\left(X^{\prime}\right) \oplus K_{X} \stackrel{i_{*}^{\prime}-p^{*}}{\rightarrow} \mathbf{R} p_{*} K_{X^{\prime}}\right)
$$

where the C.M.H.C. $K_{Y^{\prime}}\left(X^{\prime}\right)$ for cohomology of $X^{\prime}$ with support in $Y^{\prime}$ is given in $\$ 1$, defines the canonical M.H.S. on $H_{Y}^{*}(X, Z)$, the cohomology of $X$ with support on $Y$, via the projection

$$
K_{Y, X}^{t} \rightarrow T_{M} \mathbf{R} p_{*} K_{Y^{\prime}}\left(X^{\prime}\right) \underset{\operatorname{Tr} p}{\rightarrow} T \mathbf{R} \underline{\Gamma}_{Y} \mathbf{Z}_{X} .
$$

COROllary III.2.3. (i) We have long exact sequences of M.H.S.:

$$
\begin{gathered}
\rightarrow H^{i-1}(X-Y, \mathbf{C}) \rightarrow H_{Y}^{i}(X, \mathbf{C}) \rightarrow H^{i}(X, \mathbf{C}) \rightarrow H^{i}(X-Y, \mathbf{C}) \rightarrow, \\
0 \rightarrow H_{Y}^{i}(X, \mathbf{C}) \underset{p^{*+i_{*}}}{\rightarrow} H_{Y^{\prime}}^{i}\left(X^{\prime}, \mathbf{C}\right) \oplus H^{i}(X, \mathbf{C}) \underset{i_{*}^{\prime}-p^{*}}{\rightarrow} H^{i}\left(X^{\prime}, \mathbf{C}\right) \rightarrow 0 .
\end{gathered}
$$

(ii)

$$
\begin{gathered}
0 \rightarrow \mathrm{Gr}_{i}^{W} H_{Y}^{i}(X, \mathbf{C}) \rightarrow \mathrm{Gr}_{i}^{W} H_{Y^{\prime}}^{1}\left(X^{\prime}, \mathbf{C}\right) \oplus H^{i}(X, \mathbf{C}) \rightarrow H^{i}\left(X^{\prime}, \mathbf{C}\right) \rightarrow 0, \\
0 \rightarrow \mathrm{Gr}_{i}^{W} H^{i-1}(X-Y, \mathbf{C}) \rightarrow \mathrm{Gr}_{i}^{W} H_{Y}^{i}(X, \mathbf{C}) \rightarrow H^{i}(X, \mathbf{C}) \rightarrow \mathrm{Gr}_{i}^{W} H^{i}(X-Y, \mathbf{C})
\end{gathered}
$$


and

$$
\begin{gathered}
\mathrm{Gr}_{q}^{W} H_{Y}^{i}(X, \mathrm{C}) \simeq \mathrm{Gr}_{q}^{W} H_{Y^{\prime}}^{i}\left(X^{\prime}, \mathrm{C}\right) \quad \text { for } q \neq i, \\
\mathrm{Gr}_{q}^{W} H^{i-1}(X-Y, \mathrm{C}) \simeq \mathrm{Gr}_{q}^{W} H_{Y}^{i}(X, \mathrm{C}) \text { for } q>i
\end{gathered}
$$

and $\mathrm{Gr}_{q}^{W} H_{Y}^{i}(X, \mathbf{C})=0$ for $i>1$ and $q \notin[i, 2 i-2]$.

3. M.H.S. on the cohomology of a quasi-projective variety. Let $Y$ be a subvariety of a smooth and complete variety $X$. Denote by $V$ the closure of $Y$ in $X$ and $Z=V-Y$ the border of $Y$, and consider the diagram (D):

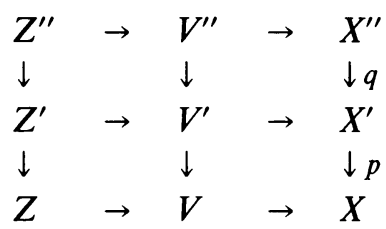

The morphism $p$ is obtained by a sequence of blowings up with centers above $Z$, such that $Z^{\prime}=p^{-1} Z$ becomes a N.C.D. in $X^{\prime}$ and $X^{\prime}-Z^{\prime} \simeq X-Z$. The variety $V^{\prime}$ is the inverse $p^{-1} V$. The morphism $q$ is obtained by successive blowings up over $V^{\prime}$ such that $V^{\prime \prime}=q^{-1} V^{\prime}$ becomes a N.C.D. in $X^{\prime \prime}$ and $X^{\prime \prime}-V^{\prime \prime} \simeq X^{\prime}-V^{\prime} \simeq X-V$. We can suppose also $Z^{\prime \prime}=q^{-1} Z^{\prime}$ is a N.C.D. in $X^{\prime \prime}$. Consider the subdiagram (D') of (D):

$$
\begin{array}{lllll}
Y^{\prime} \simeq V^{\prime \prime}-Z^{\prime \prime} & \stackrel{i^{\prime \prime}}{\rightarrow} & X^{\prime \prime}-Z^{\prime \prime} & \stackrel{j^{\prime \prime}}{\leftarrow} & X^{\prime \prime}-V^{\prime \prime} \\
& & \downarrow q & & \\
q_{Y} \downarrow & & & \downarrow q \\
Y \simeq V^{\prime}-Z^{\prime} & \stackrel{i^{\prime}}{\rightarrow} & X^{\prime}-Z^{\prime} & \leftarrow & X^{\prime}-V^{\prime}
\end{array}
$$

THEOREM III.3.1. With the previous notation, let $K_{X^{\prime \prime}}\left\langle Z^{\prime \prime}\right\rangle$ (resp. $K_{V^{\prime \prime}}\left\langle Z^{\prime \prime}\right\rangle$, $K_{X^{\prime}}\left\langle Z^{\prime}\right\rangle$ ) denote the C.M.H.C. described in I for the open smooth variety $X^{\prime \prime}-Z^{\prime \prime}$ (resp. the open N.C.D. $V^{\prime \prime}-Z^{\prime \prime}$ and the open variety $X^{\prime}-Z^{\prime}$ ). The M.H.S. on $H^{*}(Y, \mathrm{Z})$, the cohomology of $Y$, can be described by the following C.M.H.C. mixed cone:

$$
K_{Y, X}=C_{M}\left(\mathbf{R} q_{*} K_{X^{\prime \prime}-Z^{\prime \prime}} \stackrel{i^{\prime \prime *}-\operatorname{Tr} q}{\rightarrow} i_{*}^{\prime} \mathbf{R} q_{Y, *} K_{V^{\prime \prime}}\left\langle Z^{\prime \prime}\right\rangle \oplus K_{X^{\prime}}\left\langle Z^{\prime}\right\rangle\right)
$$

where the morphism $\operatorname{Tr} q$ is dual to $q^{*}: K_{X^{\prime}-Z^{\prime(\cdot)}} \rightarrow K_{X^{\prime \prime}-Z^{\prime \prime}(\cdot)}$ for cohomology with compact support, and the canonical isomorphism $q_{Y}^{*}: \mathbf{Z}_{Y} \rightarrow K_{V^{\prime \prime}}^{\mathbf{Z}}\left\langle Z^{\prime \prime}\right\rangle \rightarrow K_{Y, Z}^{\mathbf{Z}}$ gives the exact sequences of M.H.S.:

$$
\begin{aligned}
0 \rightarrow H^{i}\left(X^{\prime \prime}-Z^{\prime \prime}, \mathrm{C}\right) \stackrel{i^{\prime \prime *}-\operatorname{Tr} q}{\rightarrow} H^{i}\left(V^{\prime \prime}-Z^{\prime \prime}, \mathrm{C}\right) \\
\\
\quad H^{i}\left(X^{\prime}-Z^{\prime}, \mathrm{C}\right) \stackrel{(\operatorname{Tr} q) / Y+i^{\prime *}}{\rightarrow} H^{i}(Y, \mathrm{C}) \rightarrow 0
\end{aligned}
$$

which shows that $\mathrm{Gr}_{q}^{W} H^{i}(Y, \mathrm{C})=0$ for $q \notin[0,2 i]$.

A more elementary formulation of Theorem III.3.1 is as follows. 
THEOREM III.3.1'. With the previous notation, the canonical M.H.S. on $H^{*}(Y, \mathrm{C})$ the cohomology of $Y$, can be described by the bifiltered cone

$$
K_{Y, X}^{\mathbf{C}}=C_{M}\left(\mathbf{R} q_{*} \Omega_{X^{\prime \prime}}^{*}\left\langle Z^{\prime \prime}\right\rangle \stackrel{i^{\prime \prime *}-\operatorname{Tr} q}{\rightarrow} i_{*}^{\prime} \mathbf{R} q_{Y, *} \Omega_{\left.V^{\prime \prime} \cdot \cdot\right)}^{*}\left\langle Z^{\prime \prime}\right\rangle+\mathbf{R} \operatorname{Id}_{*} \Omega_{X^{\prime}}^{*}\left\langle Z^{\prime}\right\rangle\right)
$$

via the canonical isomorphism $q_{Y}^{*}: \mathbf{R} \Gamma(Y, \mathbf{C}) \rightarrow \mathbf{R} \Gamma\left(Y^{\prime}, \mathbf{C}\right) \rightarrow \mathbf{R} \Gamma\left(K_{Y, X}^{\mathbf{C}}\right)$. The morphism $\operatorname{Tr} q$ is defined as dual to the morphism $q^{*}: \Omega_{X^{\prime}-Z^{\prime} \cdot(\cdot)}^{*} \rightarrow \mathbf{R} q_{*} \Omega_{X^{\prime \prime}-Z^{\prime \prime} \cdot(\cdot)}^{*}$ which is defined for cohomology with compact support since $q$ is proper.

Proof. Analogous to the previous proper case. Here we use the simplicial resolution of open N.C.D. $V^{\prime \prime}-Z^{\prime \prime}$, which has been given in $§ I .5$.

Proposition III.3.2. The dual C.M.H.C. $K_{Y, X}^{t}$ to the previous cone $K_{Y, X}$ gives rise to short exact sequences

$$
0 \rightarrow H_{i}^{c}(Y) \stackrel{i_{*}^{\prime}+\left(q^{*}\right) / Y}{\rightarrow} H_{i}^{c}\left(X^{\prime}-Z^{\prime}\right) \oplus H_{i}^{c}\left(V^{\prime \prime}-Z^{\prime \prime}\right) \stackrel{i_{*}^{\prime \prime}-q^{*}}{\rightarrow} H_{i}^{c}\left(X^{\prime \prime}-Z^{\prime \prime}\right) \rightarrow 0 .
$$

4. M.H.S. on the cohomology with compact support.

Proposition III.4.1. With the previous notation, the canonical M.H.S. on $H_{Y}^{*}(X, \mathrm{C})$, the cohomology of $X$ with support on $Y$, can be described by the bifiltered cone

$$
{ }_{h} K_{Y, X}^{\mathbf{C}}=C_{M}\left(\mathbf{R} \Gamma\left(X^{\prime}, \Omega_{X^{\prime}}^{*}\left\langle Z^{\prime}\right\rangle\right) \stackrel{j^{\prime \prime *} \circ q^{*}}{\rightarrow} \mathbf{R} \Gamma\left(X^{\prime \prime}, \Omega_{X^{\prime \prime}}^{*}\left\langle V^{\prime \prime}\right\rangle\right)\right.
$$

where $q^{*}$ is induced by $q: X^{\prime \prime}-Z^{\prime \prime} \rightarrow X^{\prime}-Z^{\prime}$ and $j^{\prime \prime *}$ by $j^{\prime \prime}: X^{\prime \prime}-V^{\prime \prime} \rightarrow X^{\prime \prime}-Z^{\prime \prime}$. We get the long exact sequence of M.H.S.:

$$
\rightarrow H_{Y}^{i}(X) \rightarrow H^{i}(X-Z) \rightarrow H^{i}(X-V) \rightarrow
$$

or by Poincaré isomorphism

$$
\rightarrow H_{i}(Y) \rightarrow H_{i}(X-Z) \rightarrow H_{i}(X-V) \rightarrow
$$

Proof. Use the triangle $\mathbf{R} \Gamma_{Y}\left(X^{\prime}-Z^{\prime}\right) \rightarrow \mathbf{R} \Gamma\left(X^{\prime}-Z^{\prime}\right) \rightarrow \mathbf{R} \Gamma\left(X^{\prime}-V^{\prime}\right)$.

We have by Poincaré duality:

Proposition III.4.2. The canonical M.H.S. on $H_{c}^{*}(Y, \mathrm{C})$, the cohomology of $Y$ with compact support, can be described by the bifiltered cone

$$
{ }_{c} K_{Y, X}^{\mathbf{C}}=C_{M}\left[\mathbf{R} \operatorname{Hom}\left(\Omega_{X^{\prime \prime}}^{*}\left\langle V^{\prime \prime}\right\rangle, K_{X^{\prime \prime}}^{\prime}\right) \stackrel{\operatorname{Tr} q \circ \operatorname{Tr} j^{\prime \prime}}{\rightarrow} \mathbf{R} \operatorname{Hom}\left(\Omega_{X^{\prime}}^{*}\left\langle Z^{\prime}\right\rangle, K_{X^{\prime}}^{*}\right)\right]
$$

giving rise to the exact sequence of M.H.S.:

$$
\rightarrow H_{c}^{i-1}(Y, \mathrm{C}) \rightarrow H_{c}^{i}\left(X^{\prime \prime}-V^{\prime \prime}, \mathrm{C}\right) \rightarrow H_{c}^{i}\left(X^{\prime}-Z^{\prime}, \mathrm{C}\right) \rightarrow H_{c}^{i}(Y, \mathrm{C}) \rightarrow
$$

Proposition III.4.3. Similarly to the proper case in III.1.1 the bifiltered cone

$$
C_{M}\left(\Omega_{X^{\prime \prime}-Z^{\prime \prime(\cdot)}}^{i^{\prime \prime *}-\operatorname{Tr} q} \stackrel{\rightarrow}{\rightarrow} \Omega_{V^{\prime \prime(\cdot)}-Z^{\prime \prime(\cdot)}}^{*} \oplus \Omega_{X^{\prime}-Z^{\prime(\cdot)}}^{*}\right)
$$

gives rise to exact sequences of M.H.S.:

$$
0 \rightarrow H_{c}^{i}\left(X^{\prime \prime}-Z^{\prime \prime}, \mathrm{C}\right) \rightarrow H_{c}^{i}\left(V^{\prime \prime}-Z^{\prime \prime}, \mathrm{C}\right)+H_{c}^{i}\left(X^{\prime}-Z^{\prime}, \mathrm{C}\right) \rightarrow H_{c}^{i}(Y, \mathrm{C}) \rightarrow 0 .
$$




\section{Applications to relative cohomology.}

1. Let $Y$ be a proper variety over $\mathbf{C}$, and $i: S \rightarrow Y$ a closed embedding. To make the proof simpler, suppose $i(S)$ contains the singularities of $Y$. Consider the following diagram:

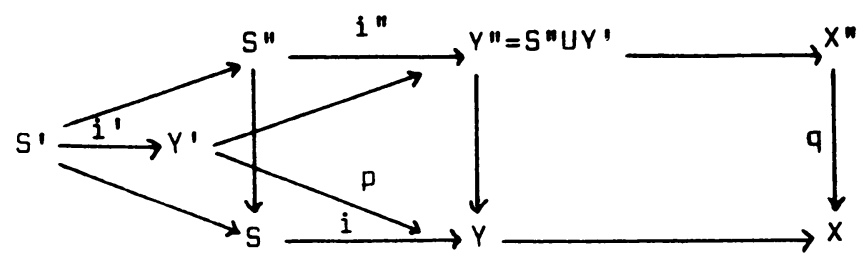

where $X$ and $X^{\prime \prime}$ are ambient smooth and complete varieties, and $q$ is obtained by successive blowing-up such that $S^{\prime \prime}$ is a N.C.D., and $Y^{\prime \prime}=q^{-1} Y$ has normal crossings and contains the desingularisation $Y^{\prime}$ of $Y$ as a component, and $S^{\prime}=S^{\prime \prime} \cap$ $Y^{\prime}$ is a N.C.D. in $Y^{\prime}$, and finally $X^{\prime \prime}-S^{\prime \prime} \simeq-S$ and $Y^{\prime}-S^{\prime} \simeq Y-S \simeq Y^{\prime \prime}-S^{\prime \prime}$. Verdier was convinced that the M.H.S. on $H_{S}^{*}(Y, \mathrm{C})$ should depend on the normal bundle of $S^{\prime}$ in $Y^{\prime}$. We want to compute the second term ${ }_{W} E_{2}$ of the spectral sequence in terms of $S^{\prime}$ and the first Chern class $C_{1}\left(N_{S_{i}^{\prime} / Y^{\prime}}\right)$ of the normal bundle of the components $S_{i}^{\prime}$ of $S^{\prime}$ in $Y^{\prime}$ in the sense of the corollary at the end of this section.

Proposition IV.1.1. With the above notation, the canonical M.H.S. can be defined on the cohomology $H_{S}^{*}(Y, Z)$ of $Y$ with support on $S$, by the M.H.C. cone

$$
C_{M}=C_{M}\left(\mathbf{R} \Gamma_{S^{\prime \prime}}\left(Y^{\prime \prime}\right) \oplus \mathbf{R} \Gamma(S) \stackrel{i^{*} \circ i_{*}^{\prime \prime}-q_{S}^{*}}{\rightarrow} \mathbf{R} \Gamma\left(S^{\prime \prime}\right)\right)[-1,-1]
$$

where $\mathbf{R} \Gamma_{S^{\prime \prime}}\left(Y^{\prime \prime}\right)$ (resp. $\mathbf{R} \Gamma(S), \mathbf{R} \Gamma\left(S^{\prime \prime}\right)$ ) denotes M.H.C. giving the M.H.S. on $H_{S^{\prime \prime}}^{*}\left(Y^{\prime \prime}\right)\left(\right.$ resp. $\left.H^{*}(S), H^{*}\left(S^{\prime \prime}\right)\right)$. The canonical isomorphism $H_{S}^{*}(Y, \mathbf{Z}) \simeq H^{*}\left(C_{M}^{\mathbf{Z}}\right)$ gives rise to the exact sequences

$$
0 \rightarrow H_{S}^{i}(Y) \underset{q_{S}^{*}+i^{*} \circ i_{*}}{\rightarrow} H_{S^{\prime \prime}}^{i}\left(Y^{\prime \prime}\right) \oplus H^{i}(S) \underset{i^{\prime \prime *} \circ i_{*}^{\prime \prime}-q_{S}^{*}}{\rightarrow} H^{i}\left(S^{\prime \prime}\right) \rightarrow 0 .
$$

Proposition IV.1.2. With the above notation, we can use the cone

$$
C_{M}^{\prime}=C_{M}\left(\mathbf{R} \Gamma_{S^{\prime}}\left(Y^{\prime}\right) \oplus \mathbf{R} \Gamma(S) \stackrel{i^{\prime *} \circ i_{*}^{\prime}-q_{S}^{*}}{\rightarrow} \mathbf{R} \Gamma\left(S^{\prime}\right)\right)[-1,-1]
$$

to get the M.H.S., on $H_{S}^{*}(Y, \mathbf{Z})$. The canonical isomorphism $H_{S}^{*}(Y, \mathbf{Z}) \simeq H^{*}\left(C_{M}^{\prime \mathbf{Z}}\right)$ gives the exact sequence

$$
\rightarrow H_{S}^{i}(Y) \stackrel{p_{S}^{*}+i^{*} \circ i_{*}}{\rightarrow} H_{S^{\prime}}^{i}\left(Y^{\prime}\right) \oplus H^{i}(S) \stackrel{i^{\prime *} \circ i_{*}^{\prime *}-q_{S}^{*}}{\rightarrow} H^{i}\left(S^{\prime}\right) \stackrel{\partial}{\rightarrow} H_{S}^{i+1}(Y) \rightarrow .
$$

Proof. As usual we work out the elementary formulation with $C_{M}^{\mathbf{C}}$ and $C_{M}^{\prime \mathbf{C}}$. The two propositions are similar, so we have included the first to get a simple proof of the naturality of the M.H.S. In fact, as one can see in the long exact sequence, the M.H.S. on $H_{S}^{i}(Y, \mathrm{C})$ is just induced by the direct $\operatorname{sum} H_{S^{\prime \prime}}^{i}\left(Y^{\prime \prime}, \mathrm{C}\right) \oplus H^{i}(S, \mathrm{C})$. This follows from the existence of a retraction of $q_{S}^{*}+i^{*} \circ i_{*}$ induced by $\operatorname{Tr} q$ : $H_{S^{\prime \prime}}^{i}\left(X^{\prime \prime}, \mathrm{C}\right) \rightarrow H_{S}^{i}(X, \mathbf{C})$, which gives rise to $\operatorname{Tr} q / Y: H_{S^{\prime \prime}}^{i}\left(Y^{\prime \prime}, \mathbf{C}\right) \rightarrow H_{S}^{i}(Y, \mathbf{C})$ such that $\operatorname{Tr} q / Y \circ q_{S}^{*}=$ Id. 
We have a natural restriction from the first cone to the second. The morphism $\partial$ in the second long exact sequence makes this diagram commutative.

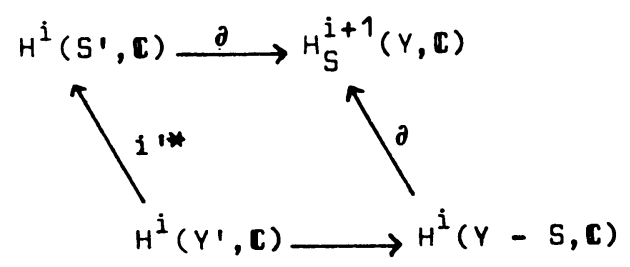

The proof of the quasi-isomorphism of each cone with $\mathbf{R} \Gamma_{S}(Y, \mathbf{C})$ is as follows:

$$
\begin{array}{ccccc}
\mathbf{R} \Gamma_{S^{\prime \prime}}\left(Y^{\prime \prime}, \mathbf{C}\right) & \stackrel{i^{\prime \prime}}{\rightarrow} & \mathbf{R} \Gamma\left(Y^{\prime \prime}, \mathbf{C}\right) & \stackrel{i^{\prime *}}{\rightarrow} & \mathbf{R} \Gamma\left(S^{\prime \prime}, \mathbf{C}\right) \\
\uparrow q_{S}^{*} & & \uparrow q^{*} & & \uparrow q_{S}^{*} \\
\mathbf{R} \Gamma_{S}(Y, \mathbf{C}) & \stackrel{i_{*}}{\rightarrow} & \mathbf{R} \Gamma(Y, \mathbf{C}) & \stackrel{i^{*}}{\rightarrow} & \mathbf{R} \Gamma(S, \mathbf{C})
\end{array}
$$

The double cone over the 4 terms of the first (resp. second) square is zero, by the exact sequences on cohomology with support in $S$ and $S^{\prime \prime}$ (resp. with compact support in $Y^{\prime \prime}-S^{\prime \prime}$ and $Y-S$ ). Then the double cone over the morphisms $i^{\prime \prime *} \circ i_{*}^{\prime \prime}$ and $i^{*} \circ i_{*}$ is itself zero. The same proof is valid if we use $Y^{\prime}$ and $S^{\prime}$ instead of $Y^{\prime \prime}$ and $S^{\prime \prime}$. Finally we give examples of resolutions to use: The complex

$$
T_{M}^{-1} C_{M}\left(\Omega_{Y^{\prime}}^{*} \rightarrow \Omega_{Y^{\prime}}^{*}\left\langle S^{\prime}\right\rangle\right) \text { in } D^{+} F_{2}\left(Y^{\prime}, \mathbf{C}\right)
$$

gives the filtrations on $\mathbf{R} \Gamma_{S^{\prime}}\left(Y^{\prime}, \mathbf{C}\right)$, then the projection on $\Omega_{Y^{\prime}}^{*}$ gives the morphism $i_{*}^{\prime}$, and the restriction to $\Omega_{S^{\prime} \cdot(\cdot)}^{*}$ the morphism $i^{\prime *}$. For $\mathbf{R} \Gamma_{S^{\prime \prime}}\left(Y^{\prime \prime}, \mathbf{C}\right)$, we use similarly $T_{M}^{-1} C_{M}\left(\Omega_{Y^{\prime} \cdot(\cdot)}^{*} \rightarrow \Omega_{Y^{\prime}}^{*}\langle S\rangle\right)$.

REMARK. It is more interesting to use the complex $\operatorname{Hom}_{S^{\prime}}\left(\Omega_{S^{\prime(\cdot)}}^{*}, K_{S^{\prime(\cdot)}}^{*}\right)$ to compute $\mathbf{R} \Gamma_{S^{\prime}}\left(Y^{\prime}, \mathbf{C}\right)$ but it should be more difficult to construct the morphism $i^{\prime *} \circ i_{*}^{\prime}$ into $\Omega_{S^{\prime}(\cdot)}^{*}$ in $C^{+} F_{2}\left(S^{\prime}, \mathrm{C}\right)$ (for this resolution).

We are not interested in describing the morphism $\mathbf{R} \Gamma(S, \mathbf{C}) \rightarrow \mathbf{R} \Gamma\left(S^{\prime \prime}, \mathbf{C}\right)$ but we have to show that it exists in $C^{+} F_{2}(\mathbf{C})$. It is easy to construct the morphism in homology:

$$
\begin{aligned}
T_{M} \mathbf{R} \Gamma_{S^{\prime \prime}}\left(X^{\prime \prime}, \mathbf{C}\right) & =\mathbf{R} \Gamma C_{M}\left(\Omega_{X^{\prime \prime}}^{*} \rightarrow \Omega_{X^{\prime \prime}}^{*}\left\langle S^{\prime \prime}\right\rangle\right) \\
C_{M}\left(\operatorname{Tr} q, \operatorname{Tr} q_{X-s}\right) & \mathbf{R} \Gamma C_{M}\left(\Omega_{X}^{*} \rightarrow \mathbf{R} p_{*} \Omega_{X^{\prime \prime}}^{*}\left\langle S^{\prime \prime}\right\rangle\right)=T_{M} \mathbf{R} \Gamma_{S}(X, \mathbf{C}) .
\end{aligned}
$$

Then we take the dual with value in $K_{X}^{*}$ (as in 3.2 .2 below).

We use the restriction on $\Omega_{S^{\prime \prime(\cdot)}}^{*} \rightarrow \Omega_{\left.S^{\prime \prime} \cdot\right)}^{*}$ to get the morphism: $\mathbf{R} \Gamma\left(S^{\prime \prime}, \mathbf{C}\right) \rightarrow$ $\mathbf{R} \Gamma\left(S^{\prime}, \mathbf{C}\right)$.

Once we construct these morphisms separately we can get the morphisms used in cones.

Remark. The M.H.C. $C_{M}^{\prime}$ defined above depends only on a neighborhood of $S$ in $Y$. The construction is the same for $S$ an algebraic variety in an analytic neighborhood $Y$. 
REMARK. The ${ }_{W} E_{1}$ terms of $C_{M}^{\mathrm{C}}$ and $C_{M}^{\prime \mathbf{C}}$ are not equal, but the ${ }_{W} E_{2}$ terms are the same.

COROLLARY IV.1.3. We have the following relations:

(1) $\operatorname{Gr}_{p}^{W} H_{S}^{i}(Y, \mathbf{C}) \simeq \operatorname{Gr}_{p}^{W}\left(H_{S^{\prime}}^{i}\left(Y^{\prime}, \mathbf{C}\right)\right) \simeq \operatorname{Gr}_{p}^{W}\left(H_{S^{\prime \prime}}^{i}\left(Y^{\prime \prime}, \mathbf{C}\right)\right) \simeq \operatorname{Gr}_{p}^{W}\left(H^{i}(Y-S, \mathbf{C})\right)$ for $p \geqslant i+1$.

(2) $0 \rightarrow \mathrm{Gr}_{p}^{W} H_{S}^{i}(Y, \mathrm{C}) \rightarrow \mathrm{Gr}_{p}^{W} H_{S^{\prime \prime}}^{i}\left(Y^{\prime \prime}, \mathrm{C}\right) \oplus \mathrm{Gr}_{p}^{W}\left(H^{i}(S, \mathrm{C})\right) \rightarrow \mathrm{Gr}_{p}^{W} H^{i}\left(S^{\prime \prime}, \mathrm{C}\right) \rightarrow 0$ is an exact sequence for $p \leqslant i$.

2. The spectral sequence for the $W$ filtration. The coefficients will be in $\mathrm{C}$. We have $\mathrm{Gr}_{i}^{W} \mathbf{R} \Gamma_{S}(Y) \simeq \mathrm{Gr}_{i}^{W} \mathbf{R} \Gamma_{S^{\prime}}\left(Y^{\prime}\right) \oplus \mathrm{Gr}_{i}^{W} \mathbf{R} \Gamma(S) \oplus T^{-1} \operatorname{Gr}_{i+1}^{W} \mathbf{R} \Gamma\left(S^{\prime}\right)$

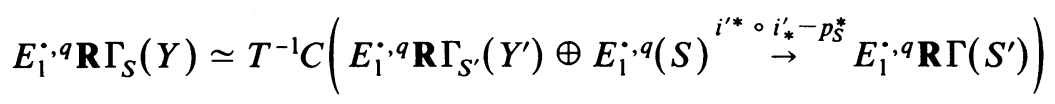

where $\left(E_{1}^{\cdot, q}(S), \delta(S)\right)$ will denote ${ }_{W} E_{1}^{\cdot, q} \mathbf{R} \Gamma(S)$. The spectral sequences of $\mathbf{R} \Gamma_{S^{\prime}}\left(Y^{\prime}\right)$ and $\mathbf{R} \Gamma\left(S^{\prime}\right)$ are given in (I); we want to make a slight modification in the middle terms of the spectral sequence as shown in the diagram:

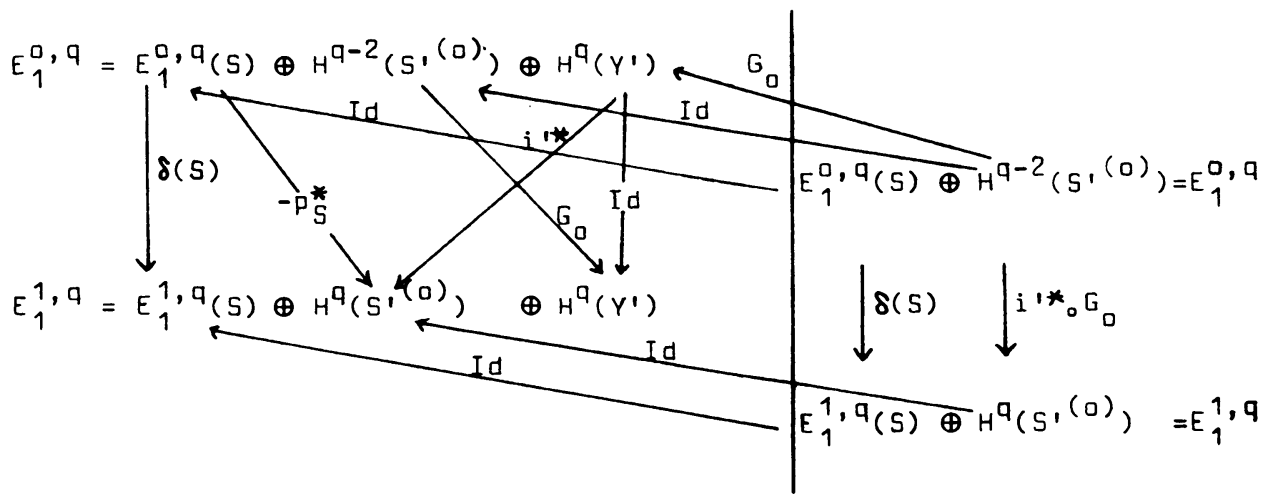

The morphism $G_{0}$ is the Gysin map of the embeddings of the components $S_{i}^{\prime}$ of $S^{\prime}$ in $Y^{\prime}$. So replacing the left side of the diagram by the right side, in the spectral sequence, we get the following quasi-isomorphic complex $E_{1}, q$ :

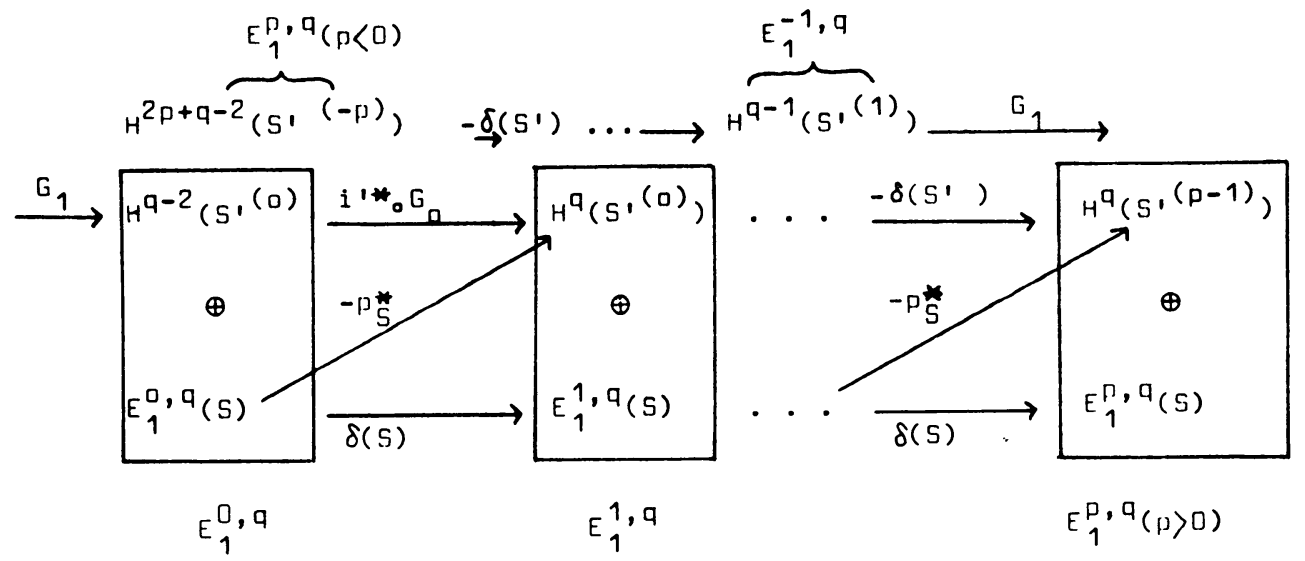


To the left, the complex resembles that of $\mathbf{R} \Gamma_{S^{\prime}}\left(Y^{\prime}\right)$, the morphism $G_{1}=G\left(\lambda_{1,1}\right)$ $-G\left(\lambda_{0,1}\right)$ is the Gysin morphism of $\lambda_{0,1}: \amalg S_{i_{0}}^{\prime} \cap S_{i_{1}}^{\prime} \rightarrow \amalg S_{i}^{\prime}\left(\right.$ resp. $\left.\lambda_{1,1}\right)$ given by the embeddings of $S_{i_{0}}^{\prime} \cap S_{i_{1}}^{\prime}$ in $S_{i_{1}}^{\prime}$ (resp. $S_{i_{0}}^{\prime}$ ). It is known that the composed morphism of the restriction $i^{\prime *}$ with the Gysin map $G_{0}: H^{q-2}\left(S^{\prime(0)}\right) \rightarrow H^{q}\left(S^{\prime(0)}\right)$ is the cup product with the first Chern class $C_{1}\left(N_{S_{i}^{\prime} / Y^{\prime}}\right)$ of the normal fiber bundle to $S_{i}^{\prime}$ in $Y^{\prime}$. The matrix of $i^{*} \circ G_{0}: \bigoplus_{i} H^{q-2}\left(S_{i}^{\prime}\right) \rightarrow \bigoplus_{i} H^{q}\left(S_{i}^{\prime}\right)$ is the dual of the intersection matrix $I: \bigoplus H_{q}\left(S_{i}^{\prime}\right) \rightarrow \bigoplus H_{q-2}\left(S_{i}^{\prime}\right)$ defined by $I\left(a_{i}\right)=a_{i} \cap_{Y^{\prime}} S_{j}^{\prime}$.

COROLlaRY IV.2.1. The cohomology of the above complex $E_{1}^{\cdot, q}$ gives the term of weight $q$ of $H_{S}^{*+q}(Y, \mathrm{C})$. In the middle term $i^{* *} \circ G_{0}$ is the dual of the intersection matrix.

3. Proposition IV.3.1. With the notation of IV1.1 the following M.H.C.

$$
\begin{aligned}
& C_{M}=C_{M}\left(\mathbf{R} \Gamma\left(Y^{\prime \prime}\right) \oplus \mathbf{R} \Gamma(S) \stackrel{i^{\prime \prime *}-q_{S}^{*}}{\rightarrow} \mathbf{R} \Gamma\left(S^{\prime \prime}\right)\right), \\
& C_{M}^{\prime}=C_{M}\left(\mathbf{R} \Gamma\left(Y^{\prime}\right) \oplus \mathbf{R} \Gamma(S) \stackrel{i^{\prime *}-p_{S}^{*}}{\rightarrow} \mathbf{R} \Gamma\left(S^{\prime}\right)\right)
\end{aligned}
$$

give, via natural quasi-isomorphism with $T \mathbf{R} \Gamma(Y)$, the long exact sequences of M.H.S.

$$
\begin{aligned}
0 \rightarrow H^{i}(Y) \rightarrow H^{i}\left(Y^{\prime \prime}\right) \oplus H^{i}(S) \rightarrow H^{i}\left(S^{\prime \prime}\right) \rightarrow 0 \\
\cdots \rightarrow H^{i}(Y) \stackrel{i^{*}+p^{*}}{\rightarrow} H^{i}\left(Y^{\prime}\right) \oplus H^{i}(S) \stackrel{i^{\prime *}-p_{S}^{*}}{\rightarrow} H^{i}\left(S^{\prime}\right) \rightarrow \cdots .
\end{aligned}
$$

The proof uses only the previous methods. We give in the Appendix the morphism $\mathbf{R} \Gamma(S) \rightarrow \mathbf{R} \Gamma\left(S^{\prime \prime}\right)$ on the complex level. The "normalisation property" as in III.1.1 applies to $C_{M}$ and explains the freedom on the choice of morphisms in the construction of the cone. This applies to $C_{M}^{\prime}$, by restriction from $C_{M}$, though it does not have normalisation.

REMARK. (1) Inductive argument. The idea in the proposition is to describe the M.H.S. of $Y$ via its desingularization. In fact we use the M.H.S. of the singular set $S$. We may use the same proposition for $S$, in an inductive argument. For simplicity, we suppose the singularity of $S$ contained in a smooth variety $Z$.

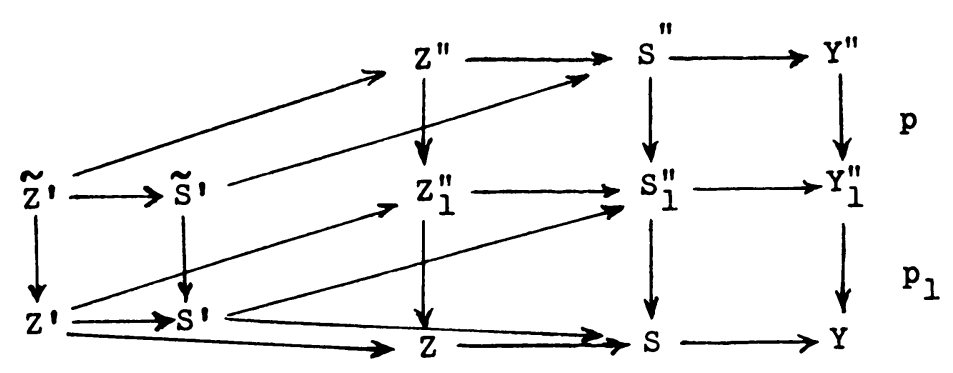

Construct the diagram; first we get the desingularization $S^{\prime}$ of $S$, embedded in $Y_{1}^{\prime \prime}$, and define $Z_{1}^{\prime \prime}=p_{1}^{-1}(Z)$ inducing on $S^{\prime}$ the N.C.D. $Z^{\prime}$, and $S_{1}^{\prime \prime}=p_{1}^{-1}(S)$. Then desingularize $Y_{1}^{\prime \prime}$ such that $S^{\prime \prime}=p^{-1}\left(S_{1}^{\prime \prime}\right), Z^{\prime \prime}=p_{1}^{-1}\left(Z_{1}^{\prime \prime}\right), \tilde{Z}=p^{-1}\left(Z^{\prime}\right)$ and $\tilde{S}^{\prime}=$ $p^{-1}\left(S^{\prime}\right)$ are all N.C.D. Then we have a resolution of $\mathbf{Z}_{Y}$ by the double cone constructed after the following scheme, and involving only N.C.D. 


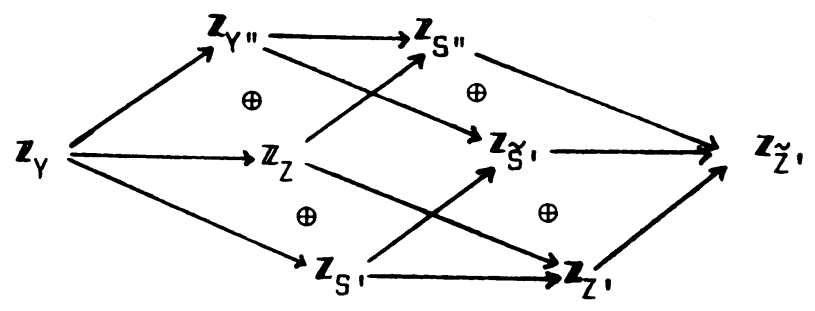

By repeating the normalization argument, we can see that the spectral sequence of the double cone gives the natural M.H.S. of $Y$.

2. The statement of the proposition involving the complex $C_{M}^{\prime}$ does not use the ambient smooth variety.

3.2. Spectral sequences.

3.2.1. With the previous notation, we give the spectral sequence of $T_{M}^{-1} C_{M}^{\prime \mathbf{C}}$ with respect to the filtration $W$.

$$
\begin{aligned}
& \mathrm{Gr}_{i}^{W}(Y)=\mathrm{Gr}_{i}^{W}\left(Y^{\prime}\right) \oplus \mathrm{Gr}_{i}^{W}(S) \oplus T^{-1} \mathrm{Gr}_{i+1}^{W}\left(S^{\prime}\right), \\
& E_{\mathrm{l}}^{\cdot, q}(Y)=T^{-1} C\left(E_{\mathrm{l}}^{\cdot, q}\left(Y^{\prime}\right)+E_{\mathrm{l}}^{\cdot}, q(S) \rightarrow E_{\mathrm{l}}^{\cdot}, q\left(S^{\prime}\right)\right) .
\end{aligned}
$$

ReMarK. The $E_{1}$ terms depend on the choice of the C.M.H.C. but the $E_{2}$ terms are natural. By an inductive argument (Remark in 3.1) we may suppose $E_{p}^{p, q}(S)=0$ for $p \notin[0, \operatorname{dim} S]$ or $q \notin[0,2 \operatorname{dim} S]$ (these conditions are not satisfied for C.M.H.C. involving the ambient space).

3.2.2. Let $R_{S}$ (resp. $R_{Y}$ ) be a C.M.H.C. giving the M.H.S. on the cohomology of $S$ (resp. $Y$ ). Suppose $R_{S}$ and $R_{Y}$ are constructed as in Remark 3.1. Then the dual ${ }^{t} R_{Y}=\mathbf{R} \operatorname{Hom}\left(R_{Y}, K_{Y}^{\cdot}\right)$ gives the M.H.S. on $H_{*}(Y)$. The spectral sequence of the bifiltered complex ${ }^{t} R_{Y}^{\mathbf{C}}=T_{M} \mathbf{R} \operatorname{Hom}\left(C_{M}^{\prime \mathbf{C}}, K_{Y}^{*}\right)$ is dual to 3.2.1:

$$
E_{1}^{p, q}\left({ }^{t} R_{Y}\right)=\left(E_{1}^{-p,-q}\left(Y^{\prime}\right) \oplus E_{1}^{-p,-q}(S) \oplus E_{1}^{-p-1,-q}\left(S^{\prime}\right)\right)^{*}
$$

where * means dual in $\mathbf{C}$.

\section{Appendix to Part II, §III.1.}

1. Functoriality.

LEMMA 1.1. Let $f: Y \rightarrow Z$ be a closed embedding of complete varieties, and $i: Z \rightarrow X$ a closed embedding in a complete and smooth variety $X$. The morphism $f^{*}: H^{*}(Z, \mathbf{C}) \rightarrow$ $H^{*}(Y, \mathrm{C})$ is a morphism of M.H.S. computed with the embeddings $i$ and $i \circ f$.

Proof. Consider the following diagram (D):

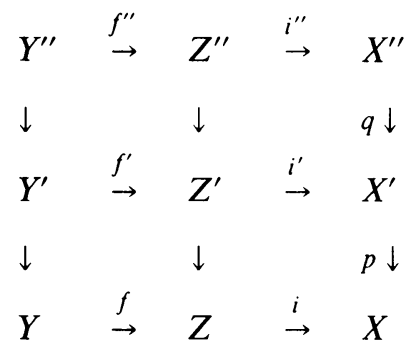


where we start by blowing up $Y$ in a N.C.D. $Y^{\prime}$ in $X^{\prime}$ and we put $Z^{\prime}=p^{-1} Z$. Then we blow up $Z^{\prime}$ in a N.C.D. $Z^{\prime \prime}$ in $X^{\prime \prime}$ such that $Y^{\prime \prime}=q^{-1} Y^{\prime}$ is also a N.C.D. in $X^{\prime}$. We have seen in II.5 that the Gysin morphisms

$$
\begin{array}{ll}
(\operatorname{Tr} q) / Y: \mathbf{R}(p \circ q) / Y, * Q_{Y^{\prime \prime}(\cdot)} \rightarrow \mathbf{R} p / Y, * Q_{Y^{\prime} \cdot()} & \text { in } D^{+} F(X, \mathbf{Q}), \\
(\operatorname{Tr} q) / Y: \mathbf{R}(p \circ q) / Y, * \Omega_{Y^{\prime \prime} \cdot(\cdot)}^{*} \rightarrow \mathbf{R} p / Y, * \Omega_{Y^{\prime} \cdot(\cdot)}^{*} & \text { in } D^{+} F_{2}(X, \mathbf{C})
\end{array}
$$

are well defined and compatible. The same is true for

$$
\operatorname{Tr} q: \mathbf{R}(p \circ q)_{*} \mathbf{Q}_{X^{\prime \prime}} \rightarrow \mathbf{R} p_{*} \mathbf{Q}_{X^{\prime}} \text { and } \operatorname{Tr} q: \mathbf{R}(p \circ q)_{*} \Omega_{X^{\prime \prime}}^{*} \rightarrow \mathbf{R} p_{*} \Omega_{X^{\prime}}^{*}
$$

We need to consider as an intermediate step the following complex:

$$
\begin{aligned}
& K_{Y^{\prime \prime}}^{\mathbf{Q}}=C_{M}\left(\mathbf{R}(p \circ q)_{*} \mathbf{Q}_{X^{\prime \prime}} \stackrel{\operatorname{Tr}(p \circ q)-\left(i^{\prime \prime} \circ f^{\prime \prime}\right)^{*}}{\rightarrow}(i \circ f)_{*} \mathbf{R}(p \circ q)_{Y, *} \mathbf{Q}_{Y^{\prime \prime} \cdot(\cdot)} \oplus \mathbf{R} \operatorname{Id}_{*} \mathbf{Q}_{X}\right), \\
& K_{Y^{\prime \prime}}^{\mathbf{C}}=C_{M}\left(\mathbf{R}(p \circ q)_{*} \Omega_{X^{\prime \prime}}^{*} \rightarrow(i \circ f)_{*} \mathbf{R}(p \circ q)_{Y, *} \Omega_{Y^{\prime \prime} \cdot(\cdot)}^{*} \oplus \mathbf{R} \operatorname{Id}_{*} \Omega_{X}^{*}\right)
\end{aligned}
$$

with usual compatibility. We may denote this data by $K_{Y^{\prime \prime}}$. Then we have morphisms in $D^{+} F(X, \mathbf{Q})$ :

$$
C_{M}^{\mathbf{Q}}\left(\mathrm{Id}, f^{\prime \prime *} \oplus \mathrm{Id}\right): K_{Z, X}^{\mathbf{Q}} \rightarrow K_{Y^{\prime \prime}}^{\mathbf{Q}} \quad \text { and } \quad C_{M}^{\mathbf{Q}}\left(\operatorname{Tr} q,(\operatorname{Tr} q)_{Y} \oplus \mathrm{Id}\right): K_{Y^{\prime \prime}}^{\mathbf{Q}} \rightarrow K_{Y, X}^{\mathbf{Q}} \text {. }
$$

The same is true in $D^{+} F_{2}(X, \mathbf{C})$. As before these constructions are compatible with the normalisation data in $D^{+}(X, \mathbf{Q})$ or $D^{+}(X, \mathbf{C})$. The only new point to check is the commutativity of the diagram:

$$
\begin{array}{ccc}
i_{*} \circ(p \circ q)_{Z, *} \mathbf{Q}_{Z^{\prime \prime}} & \underset{f^{\prime * *}}{\rightarrow}(i \circ f)_{*}(p \circ q)_{Y, *} \mathbf{Q}_{Y^{\prime \prime}} \underset{(\operatorname{Tr} q) / Y}{\rightarrow} & (i \circ f)_{*} p_{Y, *} \mathbf{Q}_{Y^{\prime}} \\
\uparrow(p \circ q)_{Z}^{*} & \stackrel{f^{*}}{\rightarrow} & \uparrow p_{Y}^{*} \\
i_{*} \mathbf{Q}_{Z} & \rightarrow & (i \circ f)_{*} \mathbf{Q}_{Y}
\end{array}
$$

which follows from the fact $(\operatorname{Tr} q) / Y \circ q_{Y}^{*}=$ Id.

REMARK. We have defined morphisms $f^{* \mathbf{Q}}: K_{Z, X}^{\mathbf{Q}} \rightarrow K_{Y, X}^{\mathbf{Q}}$ in $D^{+} F(X, \mathbf{Q})$ and $f^{* \mathbf{C}}$ : $K_{Z, X}^{\mathbf{C}} \rightarrow K_{Y, X}^{\mathbf{C}}$ in $D^{+} F_{2}(X, \mathbf{C})$ but since we have made arbitrary choices, we do not have compatibility in $D^{+} F(X, \mathbf{C})$ but only in $D^{+}(X, \mathbf{C})$.

1.2. Independence of the blowing up of $Y$ in $X$. Let $p_{1}: X_{1}^{\prime} \rightarrow X$ and $p_{2}: X_{2}^{\prime} \rightarrow X$ be two blowings up such that $p_{1}^{-1}(Y)=Y_{1}^{\prime}$ and $p_{2}^{-1}(Y)=Y_{2}^{\prime}$ are N.C.D. Then consider

$$
X_{1,2}=X_{1}^{\prime} \times_{X} X_{2}^{\prime} \stackrel{p_{1.2}}{\rightarrow} X
$$

which may be singular in $Y_{1,2}=p_{1,2}^{-1}(Y)$, and take a desingularisation $X^{\prime \prime}$ of $X_{1,2}$ such that $Y_{1,2}$ becomes a N.C.D. $Y^{\prime \prime}$ in $X^{\prime \prime}$.

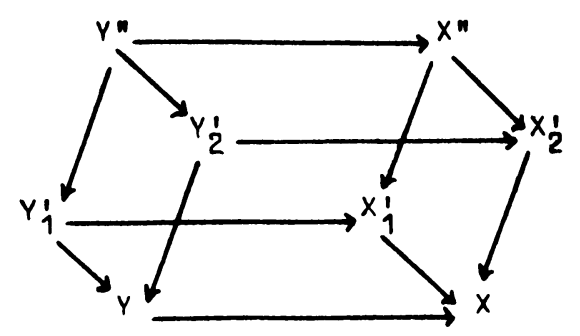


Then it will be enough to compare $\left(Y^{\prime \prime}, X^{\prime \prime}\right)$ to each $\left(Y_{i}^{\prime}, X_{i}^{\prime}\right)$. This can be done with the method above using the Gysin morphism $X^{\prime \prime} \rightarrow X_{i}^{\prime}$.

LEMMA 1.3. Let $i: Y \hookrightarrow X$ be a closed embedding in the proper smooth variety $X$ over $\mathrm{C}$, and let $Z$ be a proper and smooth variety, and $\Pi_{Y}: Y \times Z \rightarrow Y$ the first projection. The morphism $\Pi_{Y}^{*}: H^{*}(Y, \mathrm{C}) \rightarrow H^{*}(Y \times Z, \mathrm{C})$ is a morphism of M.H.S. deduced by means of the embeddings $Y \rightarrow X$ and $Y \times Z \rightarrow X \times Z$.

Proof. Consider the diagram in III.1 and its product by $Z$ as follows.

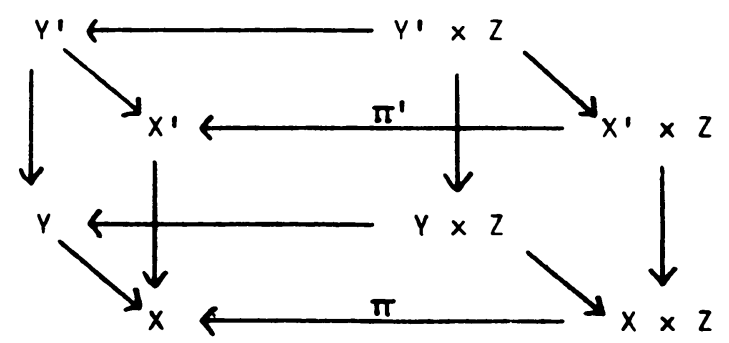

We can construct morphisms:

$$
C_{M}^{\mathbf{Q}}\left(\Pi^{* *}, \Pi_{Y}^{\prime *} \oplus \Pi^{*}\right): \Pi^{*} K_{Y, X}^{\mathbf{Q}} \rightarrow K_{Y \times Z, X \times Z}^{\mathbf{Q}} \quad \text { in } D^{+} F(X \times Z, \mathbf{Q})
$$

and the same with coefficients in $\mathrm{C}$ in the category $D^{+} F_{2}(X \times Z, \mathrm{C})$, commuting with normalisation so as to get a commutative diagram in $D^{+}(X, \mathbf{C})$.

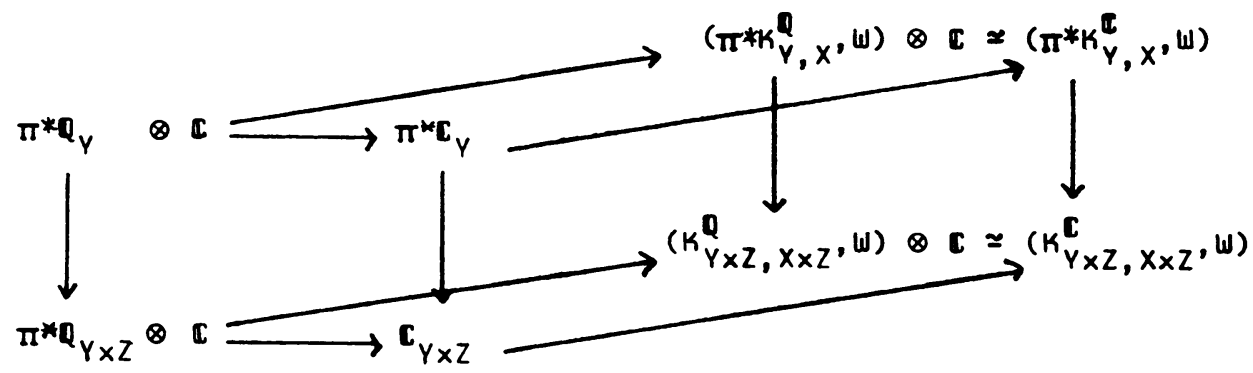

These diagrams guarantee that the different identifications necessary to get the natural morphism $H^{*}(Y, \mathrm{C}) \rightarrow H^{*}(Y \times Z, \mathrm{C})$ are induced by M.H.S. identifications since at the complex level they underlie filtered morphisms. However we get this compatibility on the cohomology level but not on the complex level.

Proposition 1.4. Let $f: Y_{1} \rightarrow Y_{2}$ be a morphism of proper varieties which can be embedded in smooth varieties. Then the natural morphism $f^{*}: H^{*}\left(Y_{2}, \mathbf{C}\right) \rightarrow H^{*}\left(Y_{1}, \mathbf{C}\right)$ underlies a morphism of M.H.S. independent of any choice. 
Proof. Let $i_{1}: Y_{1} \rightarrow X_{1}$ and $i_{2}: Y_{2} \rightarrow X_{2}$ be embeddings in proper smooth varieties. Consider the diagram:

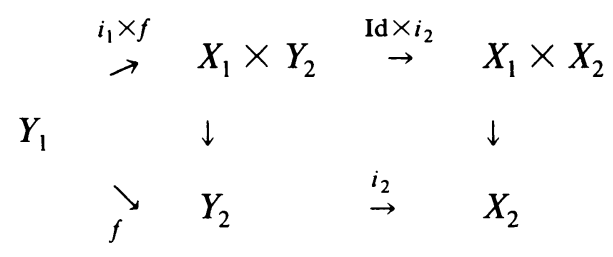

The second lemma shows that we have a morphism of M.H.S.

$$
\left(H^{*}\left(Y_{2}, \mathrm{C}\right), i_{2}\right) \rightarrow\left(H^{*}\left(X_{1} \times Y_{2}, \mathrm{C}\right), \mathrm{Id} \times i_{2}\right)
$$

where we have exhibited the embeddings used. The first lemma gives us

$$
\left(H^{*}\left(X_{1} \times Y_{2}, \mathbf{C}\right), \mathrm{Id} \times i_{2}\right) \rightarrow\left(H^{*}\left(Y_{1}, \mathbf{C}\right), i_{1} \times\left(i_{2} \circ f\right)\right) .
$$

Now we prove that the M.H.S. on $H^{*}\left(Y_{1}, \mathrm{C}\right)$ with respect to $i_{1}$ and $i_{1} \times\left(i_{2} \circ f\right)$ are compatible. Consider the diagram:

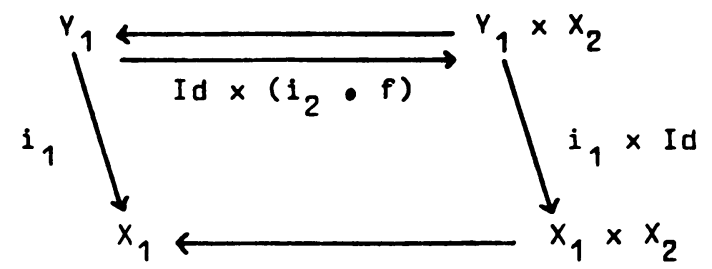

We get from the second lemma: $\left(H^{*}\left(Y_{1}, \mathbf{C}\right), i_{1}\right) \rightarrow\left(H^{*}\left(Y_{1} \times X_{2}, \mathbf{C}\right), i_{1} \times\right.$ Id $)$ and from the first lemma: $\left(H^{*}\left(Y_{1} \times X_{2}, \mathrm{C}\right), i_{2} \times \mathrm{Id}\right) \rightarrow\left(H^{*}\left(Y_{1}, \mathrm{C}\right), i_{1} \times\left(i_{2} \circ f\right)\right)$.

2. Lifting to morphism of M.H.C. In fact, for technical reasons, we are going to work in homology.

Proposition 2.1. Let $f: Y_{1} \rightarrow Y_{2}$ be a morphism of projective varieties. There exists a lifting of the morphism of M.H.S. on homology $f_{*}: H_{*}\left(Y_{1}\right) \rightarrow H_{*}\left(Y_{2}\right)$ to the M.H.C. level $f_{*}: C_{Y_{1}} \rightarrow C_{Y_{2}}$.

Proof. First we prove two lemmas as above in 1.

Lemma 2.1. With the notation of Lemma 1.1, let $K_{X}, K_{X^{\prime}-Y^{\prime}}$ and $K_{X^{\prime \prime}-Z^{\prime \prime}}$ denote the C.M.H.C. of $X, X^{\prime}-Y^{\prime}$ and $X^{\prime \prime}-Z^{\prime \prime}$. Define

$$
K_{Y}=C_{M}\left(K_{X} \rightarrow \mathbf{R} p_{*} K_{X^{\prime}-Y^{\prime}}\right) \text { and } K_{Z}=C_{M}\left(K_{X} \rightarrow \mathbf{R}(p \circ q)_{*} K_{X^{\prime \prime}-Z^{\prime \prime}}\right) \text {. }
$$

Then the morphism $\operatorname{Tr} f: K_{Y} \rightarrow K_{Z}$ is defined and induces the morphism $H_{Y}^{*}(X) \rightarrow$ $H_{Z}^{*}(X)$.

Proof. Consider the restriction $r^{*}: \mathbf{R}(p \circ q)_{*} K_{X^{\prime \prime}-Z^{\prime \prime}} \rightarrow \mathbf{R} p_{*} K_{X^{\prime}-Y^{\prime}}$ and then the trace $\operatorname{Tr} f=C_{M}\left(I_{d}, r^{*}\right)$. We must show that

$$
\begin{array}{ccccc}
K_{Y}^{\mathbf{Z}} \otimes \mathbf{Q} & \rightarrow & K_{Y}^{\mathbf{Q}} & K_{Y}^{\mathbf{Q}} \otimes \mathbf{C} & \simeq K_{Y}^{\mathbf{C}} \\
\downarrow & & \downarrow & \downarrow & \downarrow \\
K_{Z}^{\mathbf{Z}} \otimes \mathbf{Q} & \rightarrow & K_{Z}^{\mathbf{Q}} & K_{Z}^{\mathbf{Q}} \otimes \mathbf{C} & \simeq K_{Z}^{\mathbf{C}}
\end{array}
$$


and commutative diagrams in $D^{+} \mathbf{Q}$ and $D^{+} F \mathbf{C}$ respectively. We recall that the lack of commutativity comes from the fact that morphisms are defined up to homotopy. In this case, since $K_{X}^{\mathbf{Z}}=\mathbf{Z}_{X}$ and $K_{X}^{\mathbf{Q}}=\mathbf{Q}_{X}$, the morphisms are canonically defined.

LEMMA 2.2. With the notation of Lemma 1.3, define $K_{Y}$ and $K_{Y \times Z}$ as above. We have a natural isomorphism

$$
\mathbf{R} \Gamma\left(X, K_{Y}\right) \otimes \mathbf{R} \Gamma\left(Z, K_{Z}\right) \stackrel{\approx}{\rightarrow} \mathbf{R} \Gamma\left(X \times Z, K_{Y \times Z}\right)
$$

and a trace $\operatorname{Tr} \pi: \mathbf{R} \Gamma\left(X, K_{Y}\right) \otimes \mathbf{R} \Gamma\left(Z, K_{Z}\right) \rightarrow \mathbf{R} \Gamma\left(X, K_{Y}\right)$ deduced from the trace on $Z$, inducing $\operatorname{Tr} \pi: H_{Y \times Z}^{*}(X \times Z) \rightarrow H_{Y}^{*}(X)$.

Proof Continued. For any variety $V$, denote by $K_{V}$ the C.M.H.C. as in the lemmas above, and ${ }^{t} K_{V}$ its dual as explained in §II of Part II. With the notation of 1.4, we have the following morphisms of M.H.C.:

$$
\begin{array}{ccc}
\mathbf{R} \Gamma\left(X_{1} \times X_{2}, K_{Y_{1}}, i_{1} \times\left(i_{2} \circ f\right)\right) & \rightarrow & \mathbf{R} \Gamma\left(X_{1} \times X_{2}, K_{X_{1} \times Y_{2}}, \text { Id } \times i_{2}\right) \\
& \rightarrow & \mathbf{R} \Gamma\left(X_{2}, K_{Y_{2}}, i_{2}\right) .
\end{array}
$$

Still following the proof of 1.4, we have

$$
\begin{array}{ccc}
\mathbf{R} \Gamma\left(X_{1} \times X_{2}, K_{Y_{1}}, i_{1} \times\left(i_{2} \circ f\right)\right) & \rightarrow & \mathbf{R} \Gamma\left(X_{1} \times X_{2}, K_{Y_{1} \times Y_{2}}, i_{1} \times \text { Id }\right) \\
& \rightarrow & \mathbf{R} \Gamma\left(X_{1}, K_{Y_{1}}, i_{1}\right) .
\end{array}
$$

Lemma 1.2

The composition is a quasi-isomorphism; we use its inverse and we use the isomorphism given by the "independence of the blowing up" to identify

$$
\mathbf{R} \Gamma\left(X_{1} \times X_{2}, K_{Y_{1}}, i_{1} \times i_{2} \circ f\right)
$$

in the two rows.

Remark 2.3. Using the Mixed Cone of such a morphism we get a M.H.S. on the relative homology of $f$.

\section{REFERENCES}

[H II], [H III] P. Deligne, Théorie de Hodge. II, III, Publ. Math. Inst. Hautes Etudes Sci. 40 (1972), 5-57; 44 (1975), 6-77.

[E] F. Elzein, Complexe dualisant et applications, Bull. Soc. Math. France, Mémoire No. 58, 1978.

[G] P. Griffiths and W. Schmid, Recent developments in Hodge theory, Proc. Internat. Colloq. Discrete Subgroups Lie Groups (Bombay), 1973.

[V] P. Deligne (Editor), Séminaire de Géométrie Algébrique SGA 4 $\frac{1}{2}$, Lecture Notes in Math., vol. 569, Springer-Verlag, Berlin and New York, 1977 (Catégories dériveés, by J. L. Verdier, p. 262).

[VI] J. L. Verdier, Exposés VI and IX, Astérisque Nos. 36 and 37 (1976).

[VII] __ Séminaire Bourbaki 1965/66, Exposé no. 300.

[D] P. Dubois, Complexe de DeRham filtré d'une variété singulière, Bull. Soc. Math. France 109 (1981).

UeR de Mathématique, Universite Paris ViI, F-75251 Paris Cedex 5, France 